UCRL-ID-131932

\title{
MIR Wall Surveyor
}

\author{
S.K. Lehman
}

August 28, 1998

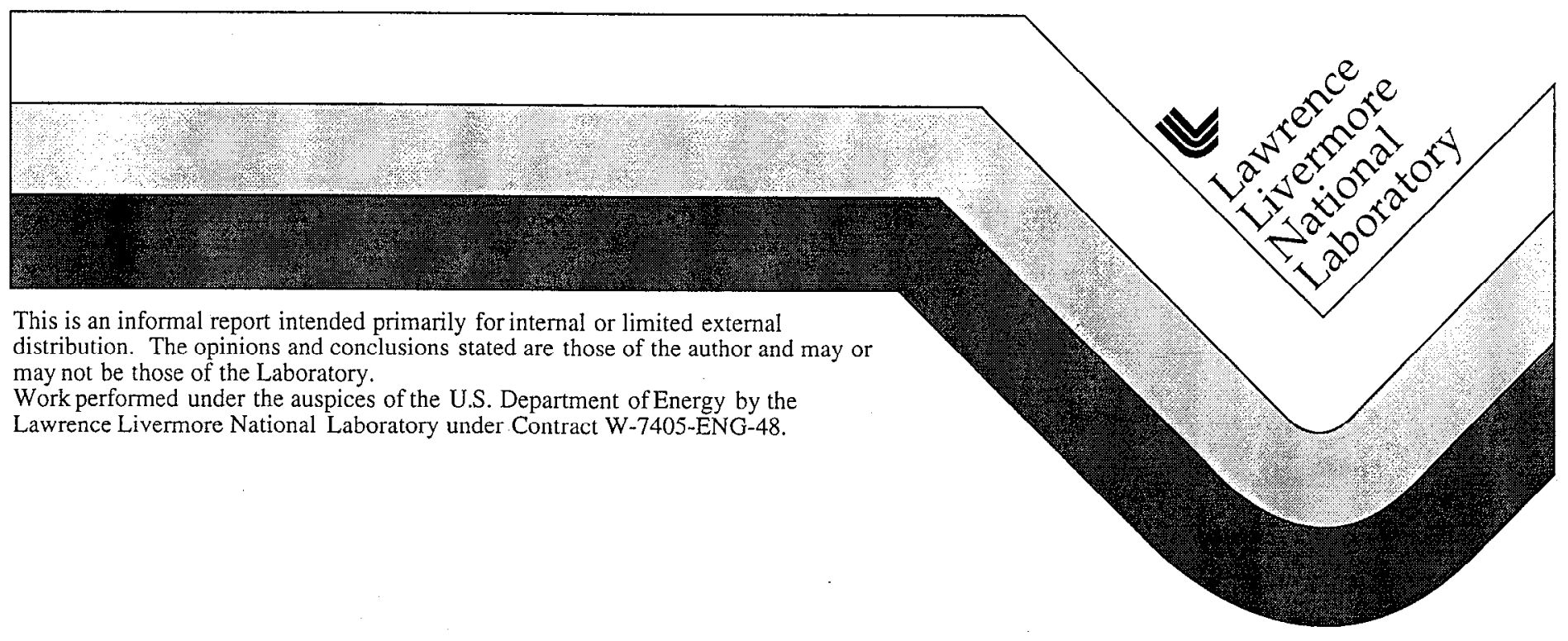




\section{DISCLAIMER}

This document was prepared as an account of work sponsored by an agency of the United States Government. Neither the United States Government nor the University of California nor any of their employees, makes any warranty, express or implied, or assumes any legal liability or responsibility for the accuracy, completeness, or usefulness of any information, apparatus, product, or process disclosed, or represents that its use would not infringe privately owned rights. Reference herein to any specific commercial product, process, or service by trade name, trademark, manufacturer, or otherwise, does not necessarily constitute or imply its endorsement, recommendation, or favoring by the United States Government or the University of California. The views and opinions of authors expressed herein do not necessarily state or reflect those of the United States Government or the University of California, and shall not be used for advertising or product endorsement purposes.

This report has been reproduced directly from the best available copy.

Available to DOE and DOE contractors from the Office of Scientific and Technical Information

P.O. Box 62, Oak Ridge, TN 37831

Prices available from (615) 576-8401, FTS 626-8401

Available to the public from the

National Technical Information Service

U.S. Department of Commerce

5285 Port Royal Rd.,

Springfield, VA 22161 


\title{
MIR Wall Surveyor
}

\author{
S. K. Lehman \\ University of California \\ Lawrence Livermore National Laboratory \\ Livermore, CA 94550
}

August 28, 1998 


\begin{abstract}
This report addresses the problem of determining the layer thickness of a wall probed with a monostatic, hand-held implementation of Lawrence Livermore National Laboratory's Micropower Impulse Radar (MIR) [1,2]. Our goal is to locate the layers of the wall, and measure its overall thickness. The physical constraints require the device to be held fixed or swept rapidly over the wall. Thus an insufficient amount of backscattered data are collected to use diffraction tomographic [3] techniques to form images. The problem is therefore one of determining the wall layers from a set of time series reflection data. We develop two channel signal processing algorithms to determine the location of the layers of a wall, using as inputs the time series returned from the wall and the incident pulse.

We study the problem using a finite difference time domain (FDTD) computer code to simulate the electromagnetic propagation within and scattering from a wall probed with five pulses. We use the results to develop and test signal processing procedures for locating the individual layers. We study two classes of algorithms: a deconvolution approach to determine a layered impulse response, and a correlation approach. After testing the algorithms on the FDTD results, we down-select to a suitable method.
\end{abstract}




\section{Contents}

1 Introduction $\quad 2$

1.1 Frequency Domain Solution .................. 5

1.2 Time Domain Solution . . . . . . . . . . . . . . . . . 6

2 Physical Model $\quad 7$

2.1 Wall Electromagnetic Propagation Simulation . . . . . . . . . . . . . 10

2.2 FDTD Simulation Results . . . . . . . . . . . . . . . . . . 14

3 Signal Processing $\quad 23$

3.1 Cross-Correlation . . . . . . . . . . . . . . . . . . . . . 30

3.2 Impulse Response Estimation . . . . . . . . . . . . . . . . . . . . 32

3.3 Energy Method . . . . . . . . . . . . . . . . . . 33

3.4 Detection Method . . . . . . . . . . . . . . . . 33

4 Results $\quad 34$

5 Application to Real Data $\quad 36$

$\begin{array}{lll}6 & \text { Conclusions } & 37\end{array}$

$\begin{array}{lll}7 & \text { Acknowledgments } & 38\end{array}$ 


\section{Introduction}

The Office of Special Technologies (OST) Wall Surveyor Radar is a hand-held monostatic implementation of Lawrence Livermore National Laboratory's (LLNL) range finder Micropower Impulse Radar (MIR) $[1,2]$. It is intended to be held fixed or rapidly swept against the surface of a wall in order to transmit and collect backscattered data. Using this reflected data, the problem under investigation is to determine the location of individual layers and overall thickness of the wall.

The constraint that the device must be held fixed or swept rapidly over the surface of the wall results in an insufficient amount of backscattered data being collected to use diffraction tomographic [3] techniques to form images. Additionally, the lack of relative antenna positioning when swept over the wall makes imaging very difficult. Thus, we reduce the complexity of the problem from that of electromagnetic reconstruction and imaging, to one of one-dimensional signal processing due to the physical constraints of the hand held MIR measurement system. The issue becomes one of determining the layers from a collection of time series of reflection data.

The MIR range finder is a fundamentally different type of radar that was invented and patented by Lawrence Livermore National Laboratory[4]. It is a pulsed radar like other ultrawideband radars, but it emits much shorter pulses than most and, because it is constructed out of a small number of common electronic components, it is compact and inexpensive.

One unique feature of the MIR is the pulse generation circuitry, which, while small and inexpensive, had never before been considered in radar applications. Each pulse is less than a billionth of a second and each MIR emits about two million of these pulses per second. Actual pulse repetition rates are coded with random noise to reduce the possibility of interference from other radars, while each is "self-tuned." Three direct advantages of the short pulse-width are:

1. With pulses so short, the MIR operates across a wider band of frequencies than a conventional radar, giving high resolution and accuracy, but also making it less susceptible to interference from other radars; 

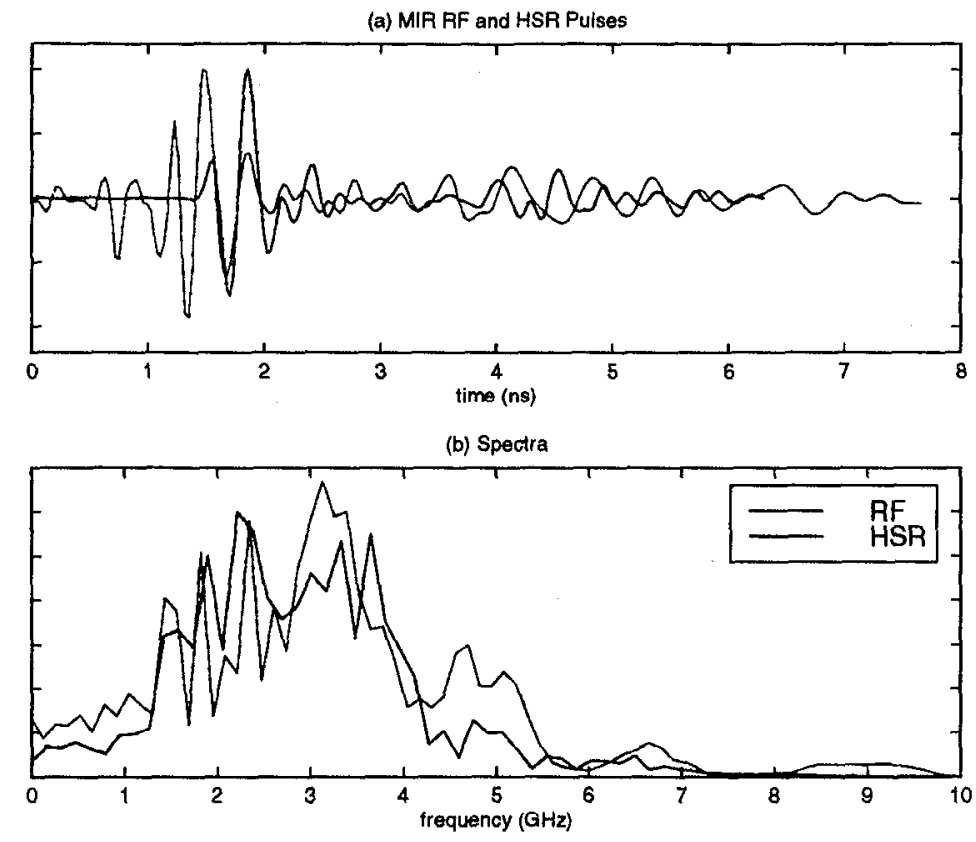

Figure 1: Two typical MIR range finder pulses: a standard range finder (RF) and a high speed radar (HSR) range finder. (a) Pulse time series. (b) Fourier spectra.

2. Since current is only drawn during this short pulse time and the pulses are infrequent, there are extremely low power requirements. For example, one type of MIR unit can operate for years on a single AA battery;

3. The microwave power emitted by the pulses is at microwatt levels and therefore medically safe.

Probably the main unique feature of this radar is the cost. The current version uses off-the-shelf electronic components so that a standard MIR board can be assembled with less than $\$ 20$ of parts.

The MIR range finder has a typical $3 \mathrm{GHz}$ bandwidth with a center frequency of $3 \mathrm{GHz}[2]$ pulse. As stated above, it operates with a pulse repetition frequency (PRF) of $2 \mathrm{MHz}$. Each pulse is range-gated back to fill in a single time bin. By sweeping the range gate over time, a complete time series is acquired. Figure 1 shows two typical MIR range finder pulses: a standard range finder (RF) and a high speed radar (HSR) range finder, along with their respective Fourier spectra. 


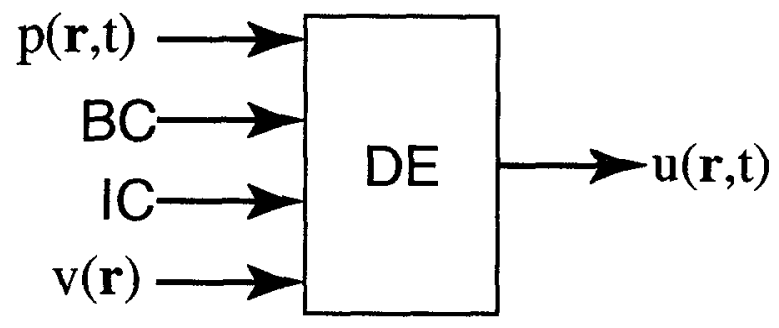

(a) Forward Problem

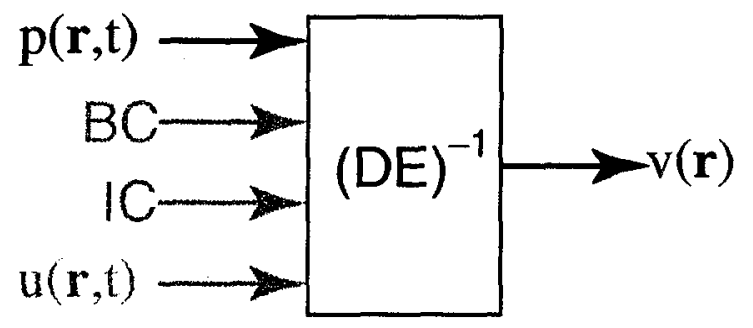

(b) Inverse Problem

Figure 2: Forward and inverse electromagnetic problems. "DE" is the differential wave equation of Eqn. 1. (a) In the forward problem, all functions are known except the field, $u(\mathbf{r}, t)$, which is to be determined. (b) In the inverse problem, the permittivity, $\epsilon(\mathbf{r})=$ $1 /\left(\mu_{0} v(\mathbf{r})\right)$, is to be determined. The IC, BC, and field may not be completely known.

The transmission and scattering of electric fields are governed by the wave equation $[5,6]$,

$$
\left[\nabla^{2}-\frac{1}{v^{2}(\mathbf{r})} \partial_{t}^{2}\right] u(\mathbf{r}, t)=p(\mathbf{r}, t)
$$

with appropriate initial conditions (IC) and boundary conditions (BC), where $\nabla^{2} \equiv \partial_{x}^{2}+$ $\partial_{y}^{2}+\partial_{z}^{2}, v(\mathbf{r})=1 / \sqrt{\mu_{0} \epsilon(\mathbf{r})}$ is the speed of light $(v(\mathbf{r})=c$ in free space), $u(\mathbf{r}, t)$ is the total electric field both in the wall and in free space, and $p(\mathbf{r}, t)$ is the transmitted pulse. We refer to this as a forward problem when all functions in Eqn. 1 are known except the field, $u(\mathbf{r}, t)$, which is to be determined.

The electrical properties of the medium are contained in the permittivity distribution, $\epsilon(\mathbf{r})$ from which we may usually infer part or all of the physical and geometric properties of the medium. When $\epsilon(\mathbf{r})$ is unknown, techniques exist to invert Eqn. 1 in order to determine it [7]. This is known as an inverse problem and is complicated by the fact the field $u(\mathbf{r}, t)$, IC, and BC are not completely known either. Figure 2 shows a graphical description of forward and inverse electromagnetic problems. The problem of using the MIR range finder to determine the layers of a wall is an inverse problem.

In the case of the MIR, assumptions must be made before attempting to solve either the forward or the inverse problem presented in Figure 2. The first is that we assume the IC are zero. This is valid since all fields have dissipated between pulses. We briefly discuss two methods for solving the forward problem, since the results serve as the basis for solving the inverse problem. The two solution methods are: frequency domain, and time domain. 


\section{$1.1 \quad$ Frequency Domain Solution}

If we Fourier transform Eqn. 1 in time, we obtain the Helmholtz equation $[5,6]$ :

$$
\left[\nabla^{2}+k^{2}(\mathbf{r}, \omega)\right] u(\mathbf{r}, \omega)=p(\mathbf{r}, \omega)
$$

where $k(\mathbf{r}, \omega) \equiv \omega / v(\mathbf{r})$ is the wavenumber, and $\omega$ is the temporal frequency of the field.

The solution to Eqn. 2 is governed by the Green's function $[5,6]$ which is the solution to:

$$
\left[\nabla^{2}+k^{2}(\mathbf{r}, \omega)\right] G\left(\mathbf{r}, \mathbf{r}^{\prime}, \omega\right)=-\delta\left(\mathbf{r}-\mathbf{r}^{\prime}\right)
$$

The Green's function, $G\left(\mathbf{r}, \mathbf{r}^{\prime}, \omega\right)$, depends upon the $\mathrm{BC}$ and problem geometry. In theory, if we had the Green's function, the inverse problem would be solved. Given the Green's function, the solution to Eqn. 2 is

$$
\begin{aligned}
u(\mathbf{r}, \omega)= & -\int_{V} d \mathbf{r}^{\prime} G\left(\mathbf{r}^{\prime}, \mathbf{r}, \omega\right) p\left(\mathbf{r}^{\prime}, \omega\right)+ \\
& \int_{S} d \mathbf{r}^{\prime}\left[G\left(\mathbf{r}^{\prime}, \mathbf{r}, \omega\right) \partial_{n} u\left(\mathbf{r}^{\prime}, \omega\right)-u\left(\mathbf{r}^{\prime}, \omega\right) \partial_{n} G\left(\mathbf{r}^{\prime}, \mathbf{r}, \omega\right)\right],
\end{aligned}
$$

where $V$ is the volume of integration, $S$ is the surface enclosing the volume, and $\partial_{n}$ is the partial derivative with respect to the surface outward normal. We have now converted the differential equation of Eqn. 2 into an integral equation. Eqn. 4 is alternatively referred to as the integral form of the Helmholtz equation or a volume integral [7].

Techniques exist to invert Eqn. 4 when large amounts of measured data are available $[3,8$, $9,10]$ and when the relative antenna positions are known. These techniques "backpropagate" the backscattered fields measured at the antennas to the scatterers, in order to form images of the scattering object being probed. In the case of the Wall Surveyor, we assume we only have a limited number of backscattered data measurements in the form of several time series and the antenna positions are not known. Fundamentally, the problem is not one of imaging, but rather determining the layers of a wall in real time. For these reasons, we eliminate the backpropagation techniques in determining the layer locations. 


\subsection{Time Domain Solution}

Assuming the IC are zero, the time domain solution to the forward problem of Eqn. 1 is given by [5]:

$$
\begin{aligned}
u(\mathbf{r}, t)= & \int_{0}^{t} d t^{\prime} \int_{V} d \mathbf{r}^{\prime} G\left(\mathbf{r}, t \mid \mathbf{r}^{\prime}, t^{\prime}\right) p\left(\mathbf{r}^{\prime}, t^{\prime}\right)+ \\
& \int_{0}^{t} d t^{\prime} \int_{S} d \mathbf{r}^{\prime}\left[G\left(\mathbf{r}, t \mid \mathbf{r}^{\prime}, t^{\prime}\right) \partial_{n} u\left(\mathbf{r}^{\prime}, t^{\prime}\right)-u\left(\mathbf{r}^{\prime}, t^{\prime}\right) \partial_{n} G\left(\mathbf{r}, t \mid \mathbf{r}^{\prime}, t^{\prime}\right)\right],
\end{aligned}
$$

where $V$ is the volume of integration, $S$ is the surface of $V, \partial_{n}$ is the partial derivative with respect to the outward normal to $S$, and $G\left(\mathbf{r}, t \mid, \mathbf{r}^{\prime}, t^{\prime}\right)$ is the Green's function of the problem which is the solution to

$$
\left[\nabla^{2}-\frac{1}{v^{2}(\mathbf{r})} \partial_{t}^{2}\right] G\left(\mathbf{r}, t \mid, \mathbf{r}^{\prime}, t^{\prime}\right)=\delta\left(\mathbf{r}-\mathbf{r}^{\prime}\right) \delta\left(t-t^{\prime}\right) .
$$

As in the last section, the Green's function depends upon the geometry of the problem and the $\mathrm{BC}$. In the time domain framework, it also depends upon the IC.

Determining the precise BC in Eqn. 5 and Green's function of Eqn. 6 is virtually impossible without some imposing two additional assumptions. The second assumption is that we may ignore the $\mathrm{BC}$. This implies we are operating in free space which, although not true for the Wall Surveyor, is acceptable in low scattering conditions. The third assumption is that we assume the Green's function can be expressed as

$$
G\left(\mathbf{r}, t \mid, \mathbf{r}^{\prime}, t^{\prime}\right)=G\left(\mathbf{r}-\mathbf{r}^{\prime}, t-t^{\prime}\right)
$$

which implies a shift invariant input/output relationship. With these assumptions, Fqn. 5 reduces to a convolution integral in space and time:

$$
u(\mathbf{r}, t)=\int d t^{\prime} \int d \mathbf{r}^{\prime} G\left(\mathbf{r}-\mathbf{r}^{\prime}, t-t^{\prime}\right) p\left(\mathbf{r}^{\prime}, t^{\prime}\right)
$$

As in the case of the frequency domain solution of Section 1.1, we have an insufficient quantity of data to invert Eqn. 8. We simplify this by making the transmitted pulse independent of space, ignoring the spatial convolution, and defining a propagation operator of time and antenna position only. We impose

$$
p\left(\mathbf{r}^{\prime}, t^{\prime}\right) \rightarrow p(t)
$$


and define

$$
h(\mathbf{r}, t) \equiv \int d \mathbf{r}^{\prime} G\left(\mathbf{r}-\mathbf{r}^{\prime}, t\right) .
$$

Thus Eqn. 8 reduces to

$$
u(\mathbf{r}, t)=\int d t^{\prime} h\left(\mathbf{r}, t-t^{\prime}\right) p\left(t^{\prime}\right) .
$$

In this form, the geometry of the wall is contained within the impulse response, $h(\mathbf{r}, t)$. In performing this simplification we have also ignored any antenna pattern as well as the volume distribution of the field within the wall and the wall complexity itself.

The problem is now that of one-dimensional deconvolution. By Fourier transforming in time Eqn. 11, the convolution in the time domain becomes a multiplication in the frequency domain [11]:

$$
U(\mathbf{r}, \omega)=H(\mathbf{r}, \omega) P(\omega),
$$

so that, in theory, the impulse response is given by

$$
h(\mathbf{r}, t)=\mathcal{F}_{\omega}^{-1}\left\{\frac{U(\mathbf{r}, \omega)}{P(\omega)}\right\},
$$

where $\mathcal{F}_{\omega}^{-1}\{\cdot\}$ is the inverse Fourier transform with respect to $\omega$.

\section{Physical Model}

In order to test the model of Eqn. 11 before collecting real data from a real wall, we developed a numerical, two-dimensional, three layer wall model which we probed using the full wave equation of Eqn. 1. The advantage of computer simulations is that they represent ideally controlled environments: noise can be non-existent or included in a known manner using thoroughly understood statistics, and there are no issues due to hardware limitations or malfunctions, or other physical limitations. The wall model is presented in Figure 3. It shows a three layer "cinder block" wall with dimensions consistent with those of an actual two- to three- block long wall. The domain consists of three regions, labeled 0,1 , and 2 . For 


\begin{tabular}{|c|l|}
\hline Region & Permittivity \\
\hline 0 & $\epsilon_{r}=1.0$ \\
1 & $\epsilon_{r}=9.0+i 0.1$ \\
2 & $\epsilon_{r}=1.0$ \\
\hline
\end{tabular}

Table 1: Permittivities of the simulation domain.

\begin{tabular}{|l|l|}
\hline Property & Value \\
\hline Physical dimensions & $96.0 \mathrm{~cm} \times 39.2 \mathrm{~cm}$ \\
Grid origin & $(-48.0,-19.6) \mathrm{cm}$ \\
Wall origin & $(-25.0,-9.6) \mathrm{cm}$ \\
Distance from xmtr/rcvr line to wall & $8.0 \mathrm{~cm}$ \\
Region 1 thickness & $3.6 \mathrm{~cm}$ \\
Region 2 thickness & $12.0 \mathrm{~cm}$ \\
\hline
\end{tabular}

Table 2: FDTD wall simulation physical dimensions.

the simulations presented here, regions 0 and 2 are air. The simulation domain permittivities are listed in Table 1. The domain physical dimensions are listed in Table 2. Figure 3 also shows a vertical slice through the center of the wall. All the simulations were multimonostatic at a fixed vertical location of $-17.6 \mathrm{~cm}$ which is a distance of $8 \mathrm{~cm}$ above the wall. That is, at each spatial location, the transmitter/receiver launched the pulse and collected the backscattered data. We anticipate reflections from each of the layers as indicated in the cross-sectional plot in Figure 3. Knowing the permittivities, we may calculate the roundtrip travel time and predict the expected single reflection or primary reflection arrival times from each of the individual layers. The travel one-way time within each layer is given by:

$$
\tau=\frac{d n}{c}=\frac{d \sqrt{\epsilon_{r}^{\prime}}}{c}
$$

where $d$ is the layer thickness, $n$ is the index of refraction, $c$ is the speed of light in a vacuum, and $\epsilon_{r}=\epsilon_{r}^{\prime}+i \epsilon_{r}^{\prime \prime}$ are the permittivities from Table 1. The "primary reflection" arrival times are computed by summing the travel times through each layer. They and are presented in Table 3 and are noted in Figure 13, and serve as a benchmark for locating the layers in the returns. 

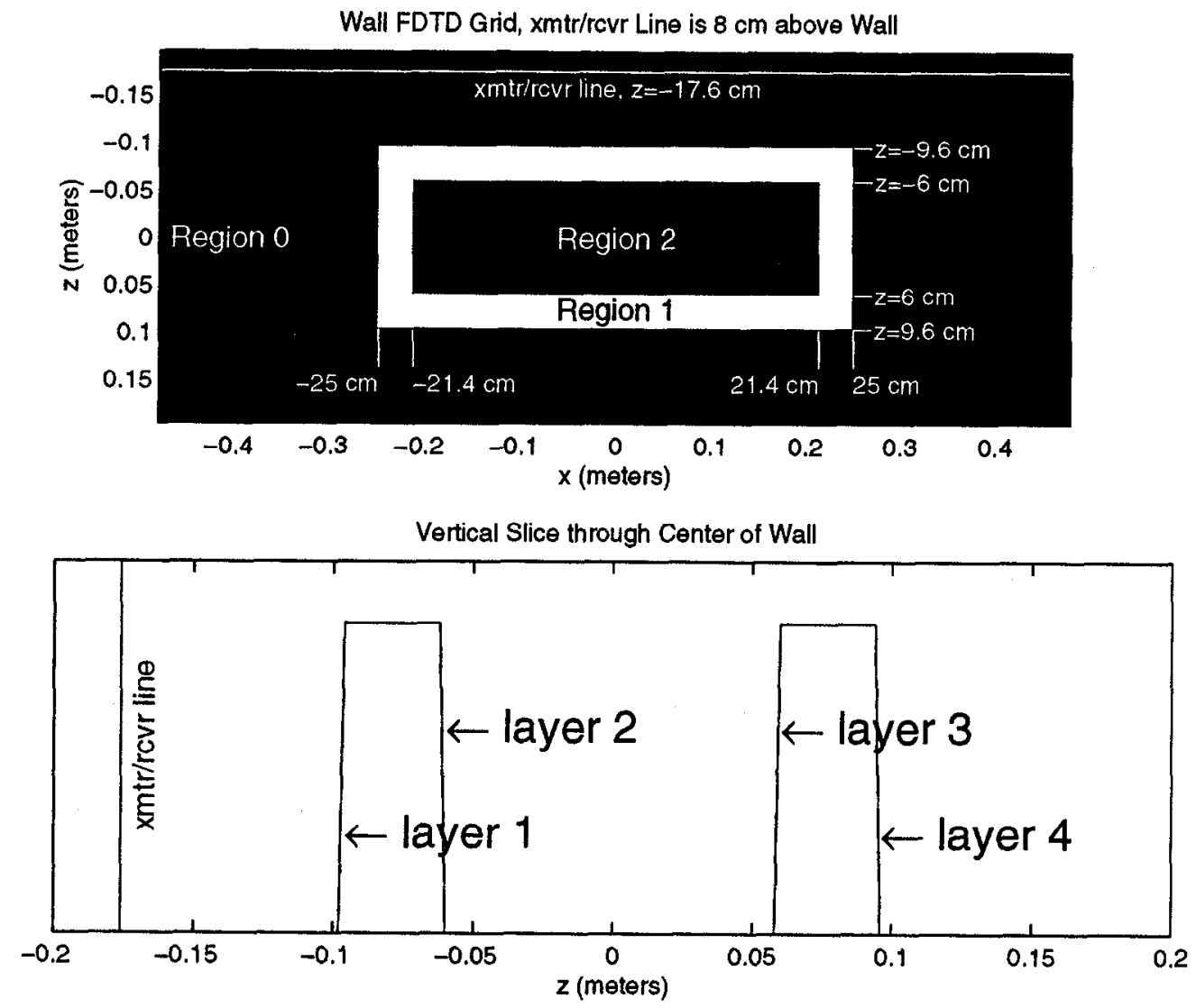

Figure 3: The electromagnetic wall propagation simulation domain. The transmitter/receiver line is indicated at the top of the image. The simulation is multimonostatic: in succession, the simulator launches a pulse and collects the backscattered field at each of the $\mathrm{xmtr} / \mathrm{rcvr}$ positions. We expect to see reflections at each of the layer interfaces as indicated. The simulation parameters are listed in Table 4.

\begin{tabular}{|llll|}
\hline Layer & $\begin{array}{l}\text { Thickness } \\
(\mathbf{c m})\end{array}$ & $\begin{array}{l}\text { One-Way } \\
\text { Travel Time (ns) }\end{array}$ & $\begin{array}{l}\text { Arrival at } \\
\text { RCVR, } \tau_{n}(\mathbf{n s})\end{array}$ \\
\hline Antenna to wall & 8.0 & 0.267 & 0.534 \\
1 (Region 1) & 3.6 & 0.360 & 1.254 \\
2 (Region 2) & 12.0 & 0.400 & 2.055 \\
3 (Region 1) & 3.6 & 0.360 & 2.775 \\
\hline
\end{tabular}

Table 3: Primary reflection receiver arrival times from each of the layers. 


\begin{tabular}{|l|l|l|l|l|l|}
\hline & \multicolumn{5}{|c|}{ Incident Pulse } \\
\hline Parameter & $\mathbf{1 8} \mathbf{~ c m ~ D O G ~}$ & $\mathbf{4} \mathbf{c m}$ DOG & 5 GHz Chirp & RF & HSR \\
\hline$L_{x}$ & $18 \mathrm{~cm}$ & $4 \mathrm{~cm}$ & $2.4 \mathrm{~m}$ & $1.5 \mathrm{~m}$ & $2.3 \mathrm{~m}$ \\
$N_{x} \times N_{z}$ & $480 \times 196$ & $960 \times 392$ & $960 \times 392$ & $480 \times 196$ & $480 \times 196$ \\
$N_{t}$ & 2000 & 7200 & 15000 & 6000 & 2500 \\
Total time & $4.69 \mathrm{~ns}$ & $3.75 \mathrm{~ns}$ & $20 \mathrm{~ns}$ & $6.39 \mathrm{~ns}$ & $12.63 \mathrm{~ns}$ \\
$\Delta x=\Delta z$ & $0.2 \mathrm{~cm}$ & $0.1 \mathrm{~cm}$ & $0.1 \mathrm{~cm}$ & $0.2 \mathrm{~cm}$ & $0.2 \mathrm{~cm}$ \\
$\Delta t$ & $2.34 \mathrm{ps}$ & $0.52 \mathrm{ps}$ & $1.33 \mathrm{ps}$ & $2.10 \mathrm{ps}$ & $2.56 \mathrm{ps}$ \\
$N_{s}$ & 91 & 91 & 11 & 91 & 91 \\
$N_{z_{0}}$ & 10 & 20 & 10 & 10 & 10 \\
\hline
\end{tabular}

Table 4: FDTD wall simulation parameters. $L_{x}$ is the free space length of pulse. $N_{x}$ is the number of samples in $x . N_{z}$ is the number of samples in $z . N_{t}$ is the total number of time steps. $N_{s}$ is the number of transmitter/receivers. $N_{z_{0}}$ is the xmtr/rcvr vertical location in pixels. The incident pulses are described in Section 2.1. DOG is derivative of a Gaussian, $\mathrm{RF}$ is the MIR range finder, and HSR is the MIR high speed radar.

\subsection{Wall Electromagnetic Propagation Simulation}

In order to understand the problem and its limitations, we investigated the underlying physics by performing several computer finite difference time domain (FDTD) $[12,13]$ simulations which numerically solve Eqn. 1 given a complete description of the wall and the incident pulse.

Explaining the details of how an electromagnetic FDTD code functions is beyond the scope of this report, however we can motivate an understanding by considering a onedimensional example. We start with the one-dimensional wave equation:

$$
\left[\partial_{x}^{2}-\frac{1}{v^{2}(x)} \partial_{t}^{2}\right] u(x, t)=p(x, t) .
$$

The goal is to discretize Eqn. 15 so that it can be implemented on a computer. The first step is to lay out a space-time grid as in Figure 4 with selected space and time intervals, $\Delta x$ and $\Delta t$, respectively.

The second step is to consider a Taylor series expansion of $u(x, t)$ about the point $\left(x_{m}+\right.$ $\left.\Delta x, t_{n}\right):$

$$
\begin{aligned}
u\left(x_{m}+\Delta x, t_{n}\right)= & u\left(x_{m}, t_{n}\right)+\Delta x \partial_{x} u\left(x_{m}, t_{n}\right)+\frac{1}{2}(\Delta x)^{2} \partial_{x}^{2} u\left(x_{m}, t_{n}\right)+ \\
& \frac{1}{6}(\Delta x)^{3} \partial_{x}^{3} u\left(x_{m}, t_{n}\right)+\frac{1}{24}(\Delta x)^{4} \partial_{x}^{4} u\left(x_{m}, t_{n}\right)+\cdots
\end{aligned}
$$




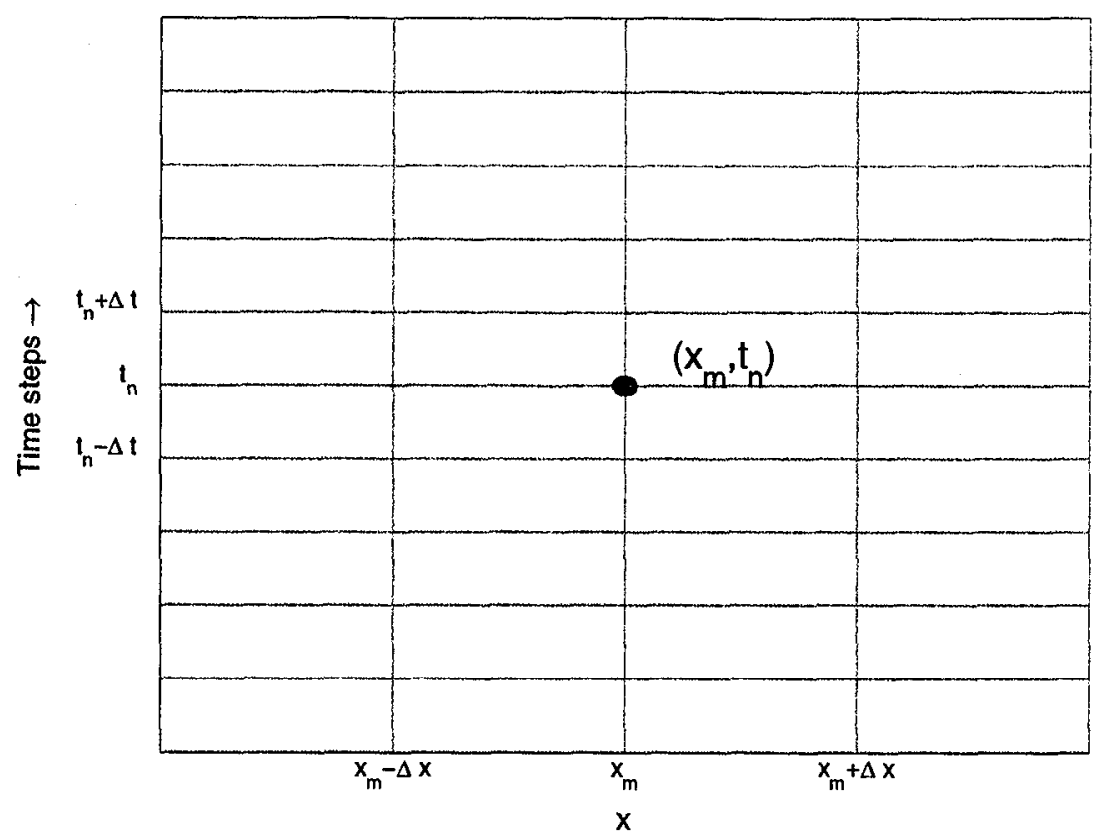

Figure 4: A finite difference time domain grid.

Similarly, consider the Taylor series expansion about the point $\left(x_{m}-\Delta x, t_{n}\right)$ :

$$
\begin{aligned}
u\left(x_{m}-\Delta x, t_{n}\right)= & u\left(x_{m}, t_{n}\right)-\Delta x \partial_{x} u\left(x_{m}, t_{n}\right)+\frac{1}{2}(\Delta x)^{2} \partial_{x}^{2} u\left(x_{m}, t_{n}\right)- \\
& \frac{1}{6}(\Delta x)^{3} \partial_{x}^{3} u\left(x_{m}, t_{n}\right)+\frac{1}{24}(\Delta x)^{4} \partial_{x}^{4} u\left(x_{m}, t_{n}\right)+\cdots
\end{aligned}
$$

Adding Eqns 16 and 17, we obtain

$$
\begin{aligned}
u\left(x_{m}+\Delta x, t_{n}\right)+u\left(x_{m}-\Delta x, t_{n}\right)= & 2 u\left(x_{m}, t_{n}\right)+(\Delta x)^{2} \partial_{x}^{2} u\left(x_{m}, t_{n}\right)+ \\
& \frac{1}{12}(\Delta x)^{4} \partial_{x}^{4} u\left(x_{m}, t_{n}\right)+\cdots
\end{aligned}
$$

Rearranging the terms in Eqn. 18 to isolate the second partial spatial derivative, we find

$$
\partial_{x}^{2} u\left(x_{m}, t_{n}\right)=\frac{u\left(x_{m}+\Delta x, t_{n}\right)-2 u\left(x_{m}, t_{n}\right)+u\left(x_{m}-\Delta x, t_{n}\right)}{(\Delta x)^{2}}+O\left[(\Delta x)^{2}\right] .
$$

Fqn. 19 states we can approximate $\partial_{x}^{2} u\left(x_{m}, t_{n}\right)$ by finite differences with an error of up to $\operatorname{order}(\Delta x)^{2}$.

We may develop a similar expression for the time derivative:

$$
\partial_{t}^{2} u\left(x_{m}, t_{n}\right)=\frac{u\left(x_{m}, t_{n}+\Delta t\right)-2 u\left(x_{m}, t_{n}\right)+u\left(x_{m}, t_{n}-\Delta t\right)}{(\Delta t)^{2}}+O\left[(\Delta t)^{2}\right] .
$$


By substituting Eqns 19 and 20 into the wave equation of Eqn. 15, we obtain

$$
\begin{gathered}
\frac{u\left(x_{m}+\Delta x, t_{n}\right)-2 u\left(x_{m}, t_{n}\right)+u\left(x_{m}-\Delta x, t_{n}\right)}{(\Delta x)^{2}}+ \\
\frac{1}{v^{2}\left(x_{m}\right)} \frac{u\left(x_{m}, t_{n}+\Delta t\right)-2 u\left(x_{m}, t_{n}\right)+u\left(x_{m}, t_{n}-\Delta t\right)}{(\Delta t)^{2}}=p\left(x_{m}, t_{n}\right) .
\end{gathered}
$$

Solving for the most current (in time) value of $u$ at the spatial point $x_{m}$, we find

$$
\begin{aligned}
u\left(x_{m}, t_{n}+\Delta t\right)= & \left(\frac{v\left(x_{m}\right) \Delta t}{\Delta x}\right)^{2}\left[u\left(x_{m}+\Delta x, t_{n}\right)-2 u\left(x_{m}, t_{n}\right)+u\left(x_{m}-\Delta x, t_{n}\right)\right] \\
& +2 u\left(x_{m}, t_{n}\right)-u\left(x_{m}, t_{n}-\Delta t\right)+v^{2}\left(x_{m}\right) p\left(x_{m}, t_{n}\right) .
\end{aligned}
$$

This is an explicit, second-order accurate (in both time and space) expression for $u\left(x_{m}, t_{n}+\right.$ $\Delta t$ ), since all quantities on the right hand side the equation are known from either the previous time step, or the IC. Although not serious in the one-dimensional case, there are mathematical issues in correctly handling the $\mathrm{BC}$ which, in free space, must absorb the fields incident on the boundary. We refer the reader to the topic of absorbing $\mathrm{BC}$ in [13] and [14]. Finally, there is an issue of numerical stability. For stable solutions, $\Delta x$ and $\Delta t$ cannot be arbitrarily chosen but must be related by[13]:

$$
\frac{\Delta x}{\Delta t} \leq v(x),
$$

that is, the sample intervals must be chosen so that the numerical propagation of the field $(\Delta x / \Delta t)$ is less than the elcctromagnetic propagation within the medium. This relationship can be derived from the Nyquist sampling theorem [15]: if $\lambda(x)$ and $f$ are, respectively, the shortest spatial wavelength and highest temporal frequency, we need, in order to sample the field without aliasing,

$$
\begin{aligned}
\Delta x & \leq \frac{\lambda(x)}{2}, \text { and } \\
\Delta t & \leq \frac{1}{2 f} .
\end{aligned}
$$

Combining Eqns 24 and 25, results in

$$
\frac{\Delta x}{\Delta t} \leq \lambda(x) f=v(x) .
$$


We ran five wall simulations using an undocumented, in-house two-dimensional FDTD $\operatorname{code}^{1}$. It models a linear multimonostatic array of transmitter/receiver pairs with fixed vertical offset from the wall. In turn and at each horizontal position, the transmitter/receiver launches the user specified pulse, $p(t)$, and records the backscattered field. The wall properties and transmitter/receiver location were identical in each simulation. The incident pulses, $p(t)$, however, were different. The five selected pulses were:

- An MIR range finder (RF) pulse digitized from a calibration data set as shown in Figure 1;

- An MIR high-speed radar (HSR) pulse digitized from a calibration data set as shown in Figure 1,

- A spatially long, with respect to the dimensions of the wall, derivative of a Gaussian (DOG) pulse of $18 \mathrm{~cm}$;

- A $5 \mathrm{GHz}$ bandwidth chirp; and,

- A spatially short DOG pulse of $4 \mathrm{~cm}$.

The DOG wave form is described by

$$
p(t)=-\left(\sigma e^{1 / 2}\right) \frac{t}{\sigma^{2}} e^{-\left(t-t_{0}\right)^{2} /\left(2 \sigma^{2}\right)}
$$

where $\sigma=0.075 \mathrm{~ns}$ for the $18 \mathrm{~cm}$ pulse, and $\sigma=0.0166782 \mathrm{~ns}$ for the $4 \mathrm{~cm}$ pulse. The 5 $\mathrm{GHz}$ chirp is governed by

$$
p(t)=\sin \left(2 \pi\left(f_{0}+a t / 2\right) t\right)
$$

where

$$
\begin{aligned}
f_{0} & =2.5 \mathrm{GHz}, \text { and } \\
a & =1.25 \mathrm{GHz}^{2} .
\end{aligned}
$$

\footnotetext{
${ }^{1}$ The code, simply called " $f d t d$ ", is a two-dimensional code written by Dr. J. E. Mast.
} 
Figure 5 shows plots of each of the five incident pulses as a function of time, space, along with their respective spectra. The $18 \mathrm{~cm}$ DOG was chosen because it approximately covers the same spectral region as the two MIR pulses. It is a reasonable model of the actual MIR pulses although the latter have a more significant amount of ringing. A detailed plot, comparing only the MIR pulses with the $18 \mathrm{~cm}$ DOG is shown in Figure 6 . The short, $4 \mathrm{~cm}$, DOG was chosen since it is spatially short enough so that its "primary reflection" returns from the individual layers are easily discerned without any signal processing. This serves as a sanity check for any signal processing algorithm. The chirp was chosen because its autocorrelation approximates an impulse and is thus ideal for a cross-correlation approach in identifying the layers in a pulse-echo environment.

Using the results from the simulated environment, we developed a signal processing algorithm to determine the locations of the layers of a three layer cinder block wall using the one-dimensional temporal convolution model of Eqn. 11. The point being if we were unable to determine the layers in the ideal case, it would be pointless to examine real data collected from a physical cinder block wall.

\subsection{FDTD Simulation Results}

The results of the FDTD simulations are presented in Figures 7 to 11. Each of these figures consist of a time series graph at the top and two images at the bottom. The images represent the solution to the wave equation of Eqn. $1, u(x, z, t)$ at a fixed $z$-offset from the wall, $z_{0}$. In terms of the solution of Eqn. 5:

$$
\begin{aligned}
u\left(x, z_{0}, t\right)= & \int_{0}^{t} d t^{\prime} \int d x^{\prime} \int d z^{\prime} G\left(x, z_{0}, t \mid x^{\prime}, z^{\prime}, t^{\prime}\right) p\left(x^{\prime}, z^{\prime}, t^{\prime}\right)+ \\
& \int_{0}^{t} d t^{\prime} \int_{S} d \Omega\left(x^{\prime}, z^{\prime}\right)\left[G\left(x, z_{0}, t \mid x^{\prime}, z^{\prime}, t^{\prime}\right) \partial_{n} u\left(x^{\prime}, z^{\prime}, t^{\prime}\right)-\right. \\
& \left.u\left(x^{\prime}, z^{\prime}, t^{\prime}\right) \partial_{n} G\left(x, z_{0}, t \mid x^{\prime}, z^{\prime}, t^{\prime}\right)\right]
\end{aligned}
$$

where we set $\mathbf{r}=\left(x, z_{0}\right)$ and $d \Omega\left(x^{\prime}, z^{\prime}\right)$ is a differential element of the volume surface area.

The images on the left show the raw simulation output, $u\left(x, z_{0}, t\right)$. The large returns across the top of the images are due to transmitter/receiver antenna coupling. We refer 


\section{Comparison of Simulation Pulses}

Time

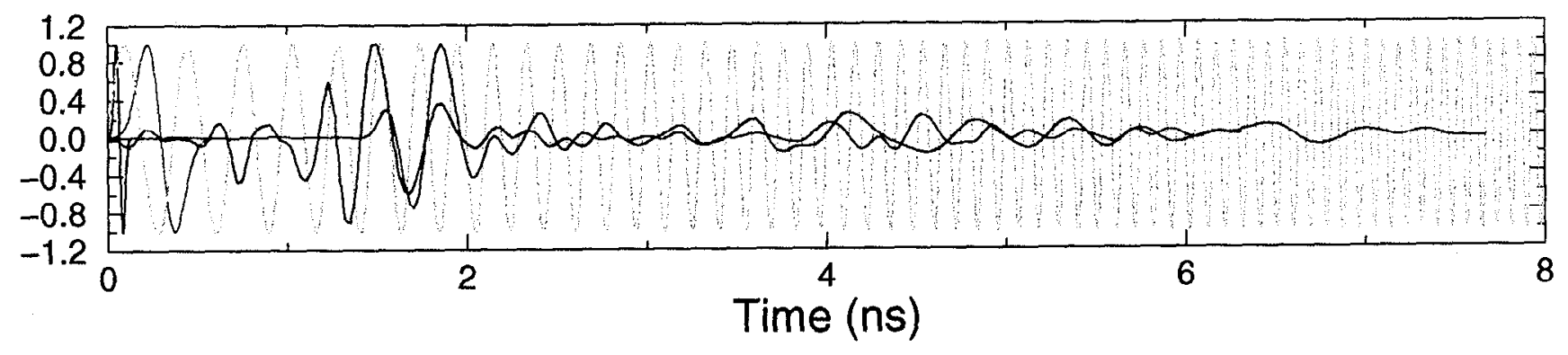

Free Space
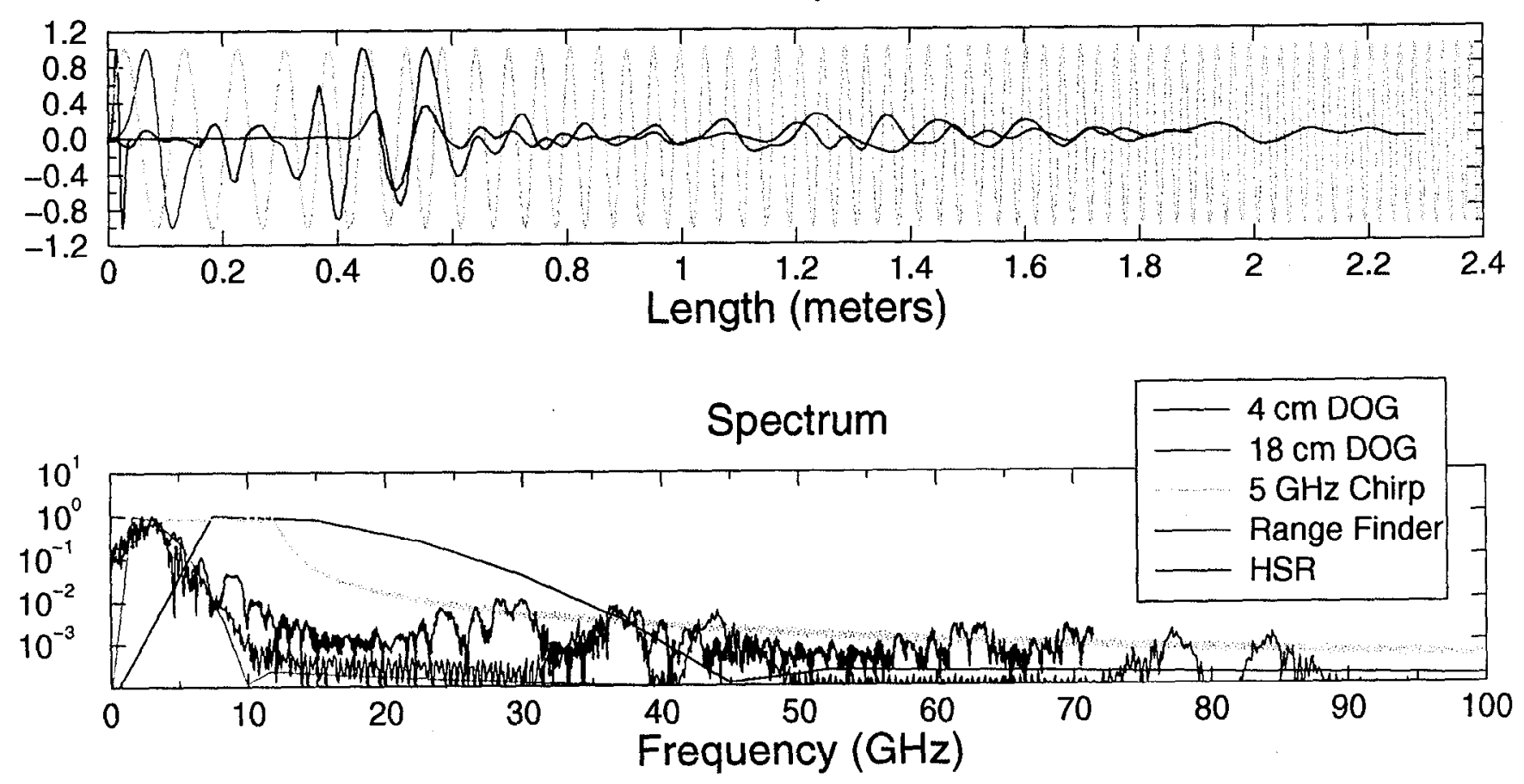

Figure 5: The five FDTD simulation pulses shown as a function of time, their free-space pulse length, and their spectra. 


\section{Comparison of MIR and $18 \mathrm{~cm} \mathrm{DOG} \mathrm{Pulses}$}

Time Domain

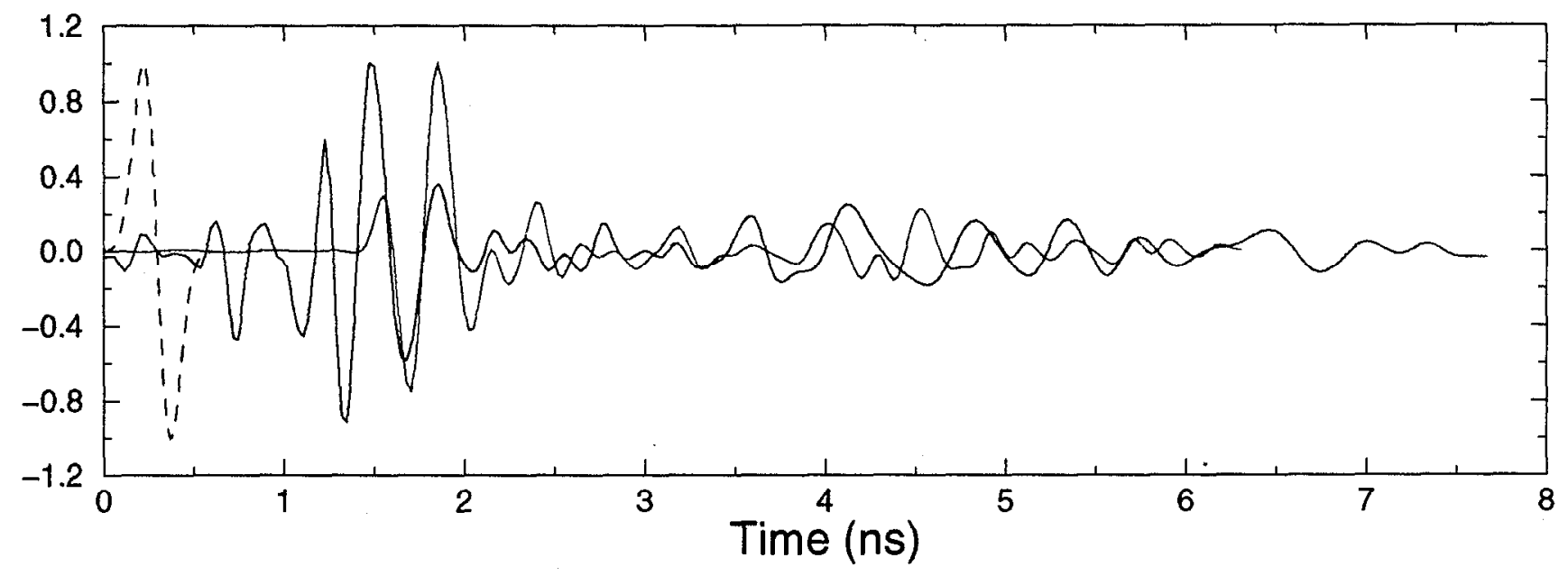

Spectra

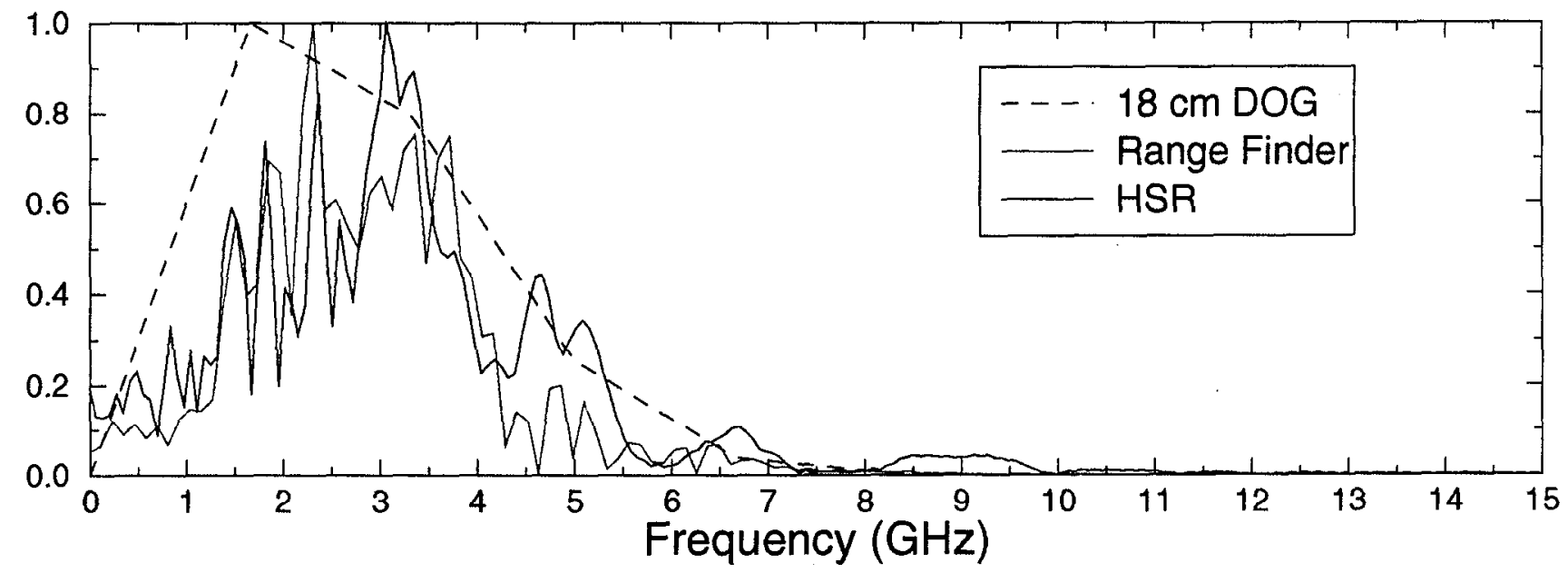

Figure 6: Comparison between the two digitized MIR pulses extracted from calibration data sets and the $18 \mathrm{~cm}$ DOG pulse. Spectrally, the DOG approximately covers the same region as the MIR pulses, however, temporally, the DOG only models the first cycle of the MIR pulses with no ringing. 
to this as the "bang." We remove it by averaging over space, five to ten time (vertical) returns on the left hand side of the image (where the scattering from the wall is negligible) and subtracting this from the whole image. This technique is known jointly as either "subaveraging" or "de-banging," and the resulting image is call the "de-banged" image. The right images show the de-banged results.

Note: In the actual Wall Surveyor which operates with a monostatic antenna, the "bang" in the data is due to the reflection from the surface of the wall. This can be easily removed since the antenna has fixed spacer of known offset in front of it. Thus the "bang" can be removed knowing the time of flight and incident pulse length.

We extracted the center return from each of the "de-banged" results and used these five time sequences as our test cases. In terms of our model in Eqn. 11, the extracted center return is

$$
r(t) \equiv u\left(x_{0}, z_{0}, t\right)=\int d t^{\prime} h\left(x_{0}, z_{0}, t-t^{\prime}\right) p\left(t^{\prime}\right),
$$

where we set $\mathbf{r}=\left(x_{0}, z_{0}\right)$, the $\mathrm{xmtr} / \mathrm{rcvr}$ location above the center of the wall.

It is clear from the results of Figures 7, 8, and 9 (the RF, HSR, and $18 \mathrm{~cm}$ DOG pulses, respectively) we are unable to discern the primary reflection returns from the layers in the center return, $r(t)$ (top graph). This is due to the free-space length of the pulse which is longer than the wall, the ringing, and the reverberation due to multiple reflections. These factors contribute to the occluding of the primary reflection returns. Figure 10 shows the chirp results. Because of its long, ringing nature, the returns from the individual layers are completely lost in the FDTD center return, $r(t)$. The layers are easily identified in the $4 \mathrm{~cm}$ DOG pulse plots of Figure 11. Although not a problem with the short (4 cm DOG) pulse the reverberations are seen as the damped oscillations in the time series.

All five cases reveal an issue with attenuation: there is insufficient power to measure the reflections from the deeper layers. Only in the $4 \mathrm{~cm}$ DOG case can the third layer be located without any signal processing. Next we discuss the signal processing techniques required to estimate the wall layer locations and overall wall thickness. 
Transmitted RF Pulse and Normalized Center Return

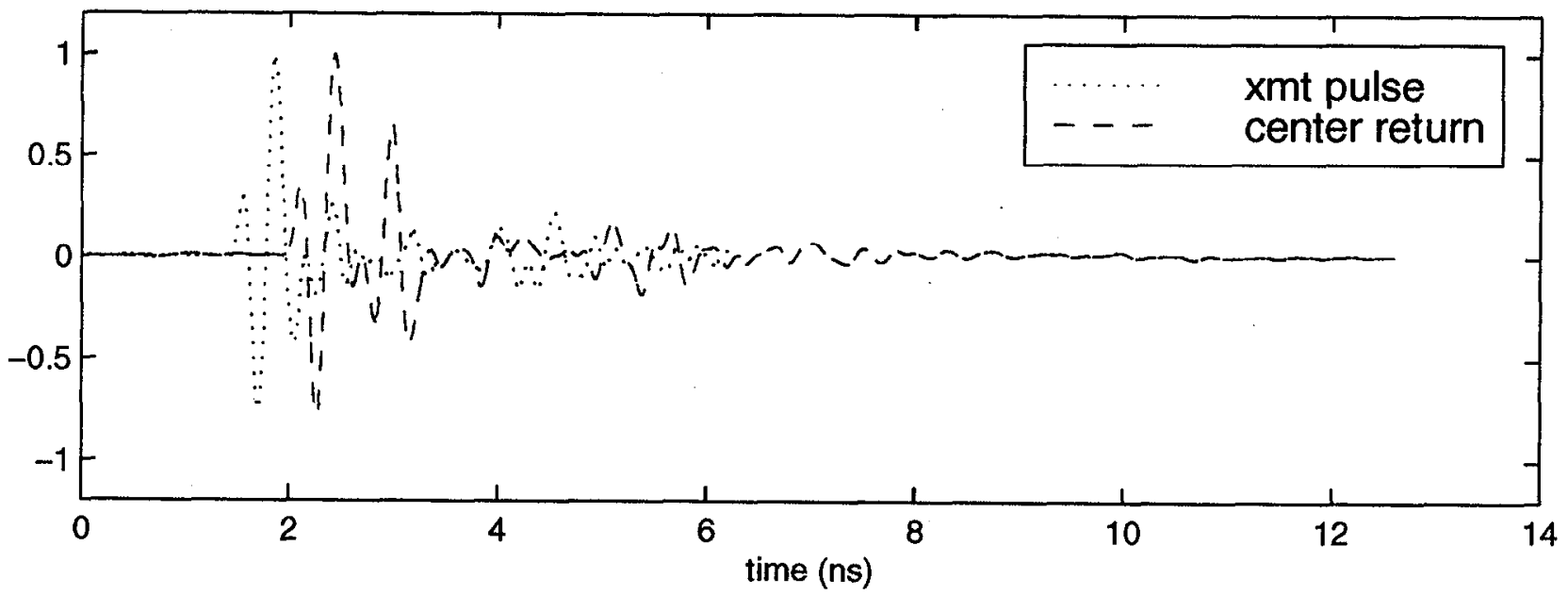

FDTD Result

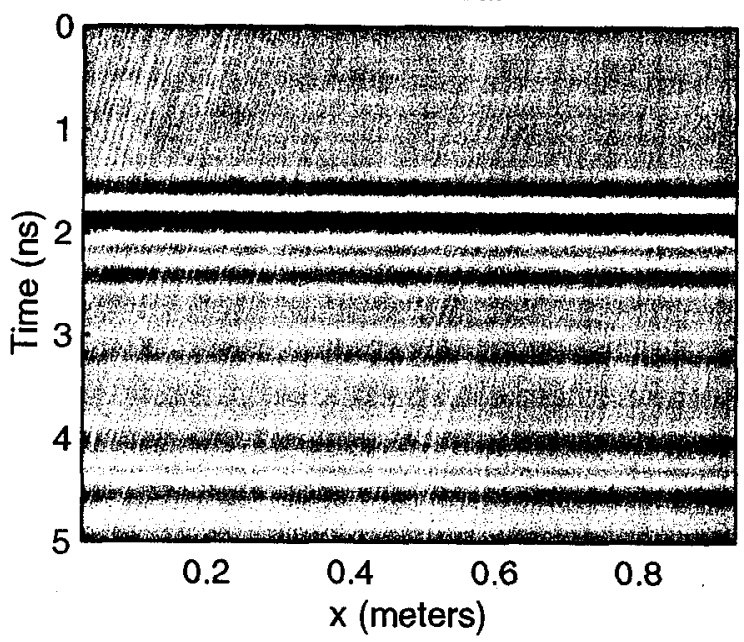

FDTD Result - Debanged

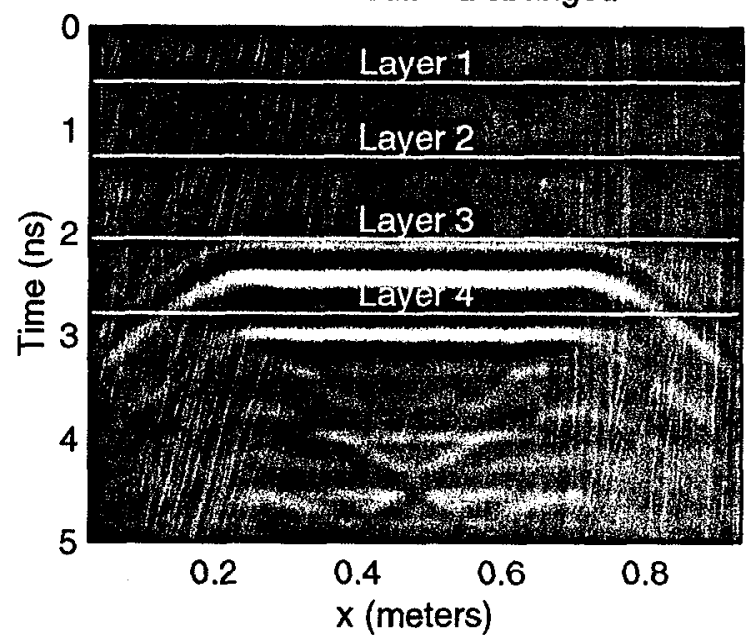

Figure 7: Range finder FDTD simulation results. The top plot shows the transmitted pulse and the return extracted from the center of the de-banged result. The left image is the raw code output. The right images is the de-banged result, that is, with the large antenna-toantenna coupling pulse removed. The de-banged image shows the location of each layer as computed with Eqn. 14. The simulation parameters are listed in Table 4. 
Transmitted HRS Pulse and Normalized Center Return

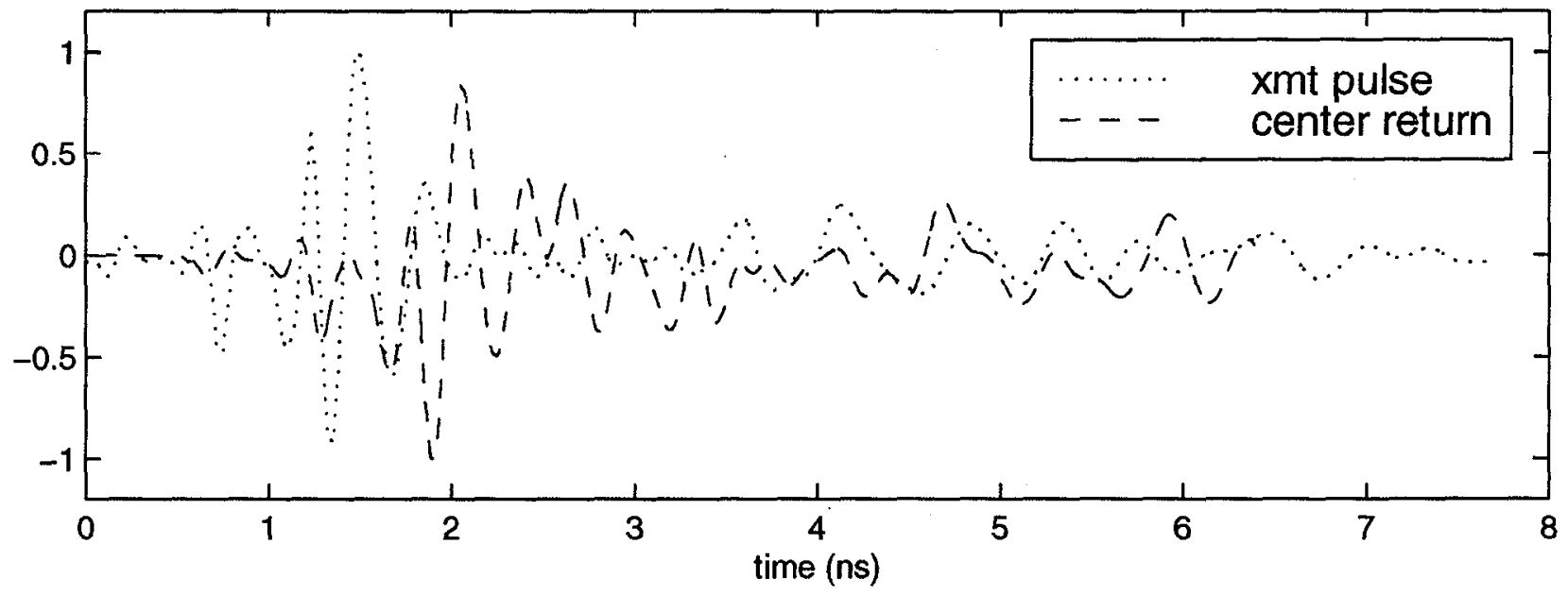

FDTD Result

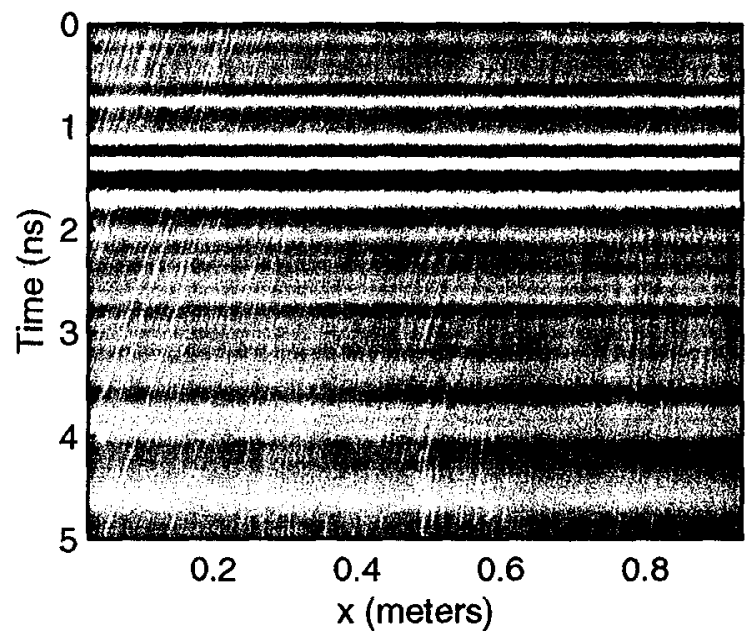

FDTD Result - Debanged

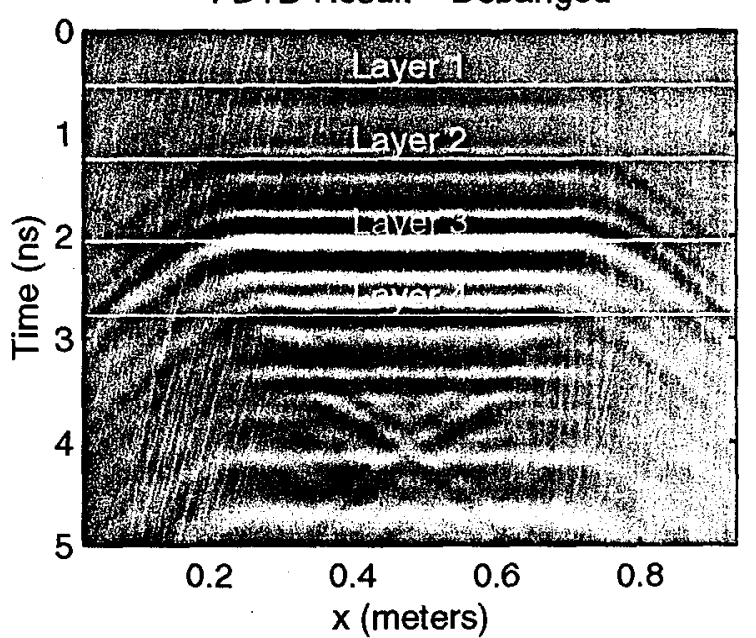

Figure 8: High speed radar FDTD simulation results. The top plot shows the transmitted pulse and the return extracted from the center of the de-banged result. The left image is the raw simulation output. The right images is the de-banged result, that is, with the large antenna-to-antenna coupling pulse removed. The de-banged image shows the location of each layer as computed with Eqn. 14. The simulation parameters are listed in Table 4. 
Transmitted $18 \mathrm{~cm}$ DOG and Normalized Center Return

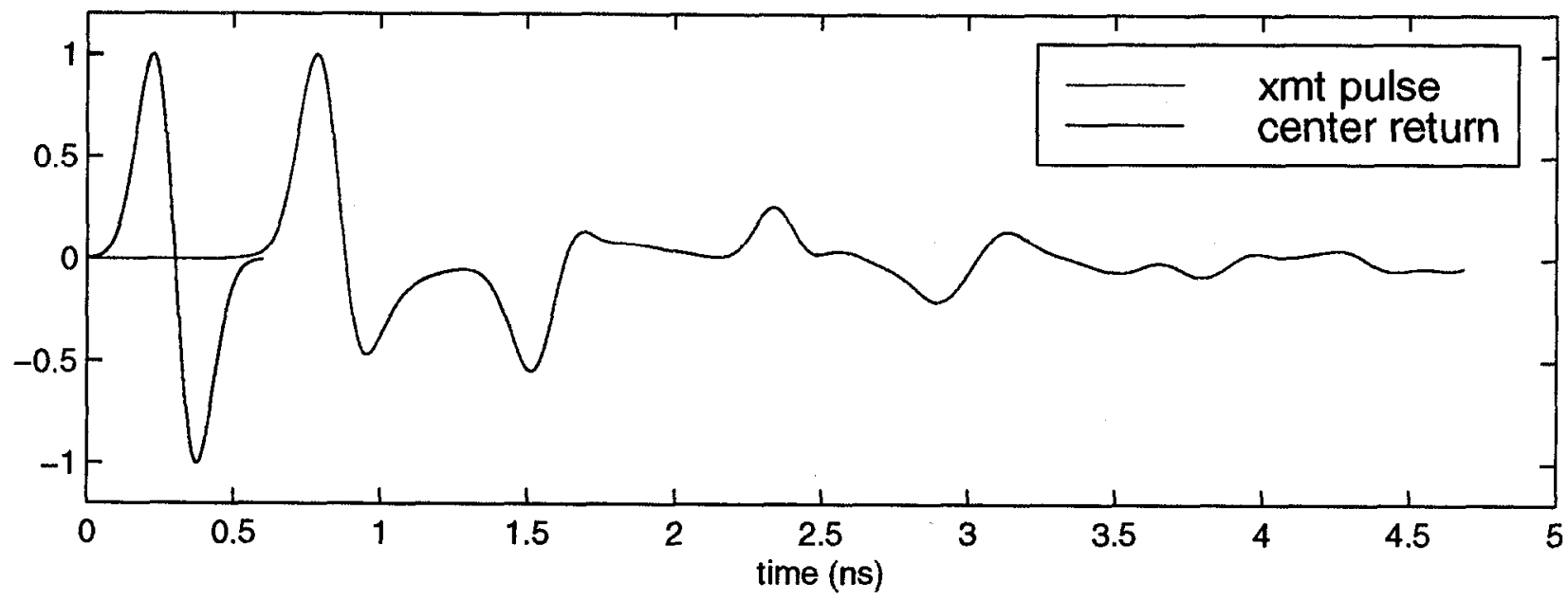

FDTD Result

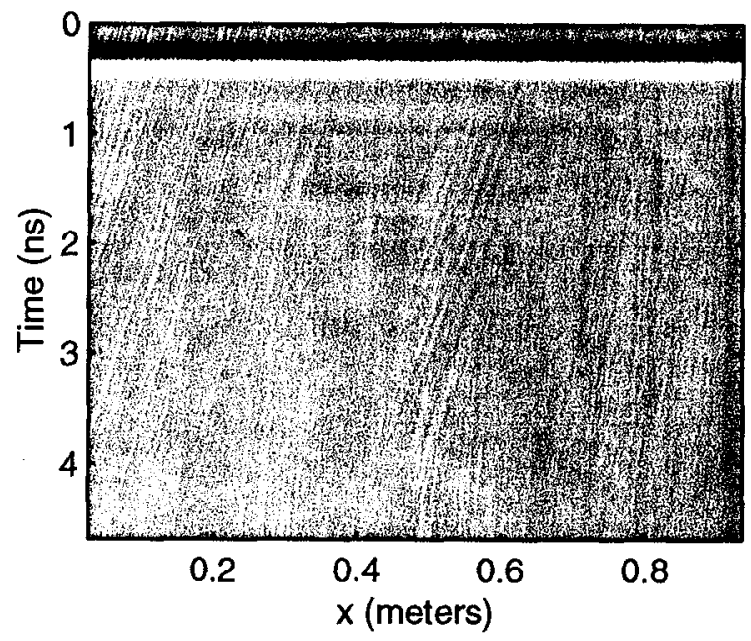

FDTD Result - Debanged

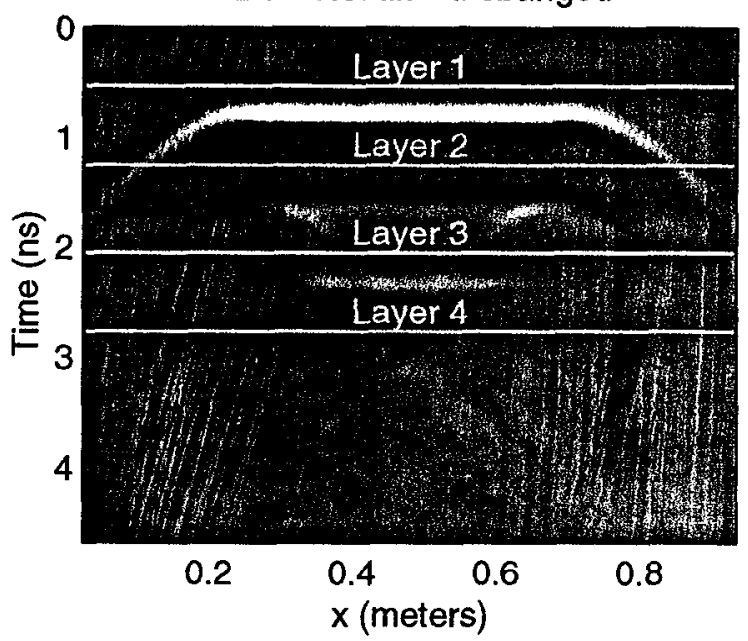

Figure 9: $18 \mathrm{~cm}$ DOG FDTD simulation results. The top plot shows the transmitted pulse and the return extracted from the center of the de-banged result. The left image is the raw simulation output. The right images is the de-banged result, that is, with the large antennato-antenna coupling pulse removed. The de-banged image shows the location of each layer as computed with Eqn. 14. The simulation parameters are listed in Table 4. 
Transmitted $5 \mathrm{GHz}$ Chirp and Normalized Center Return
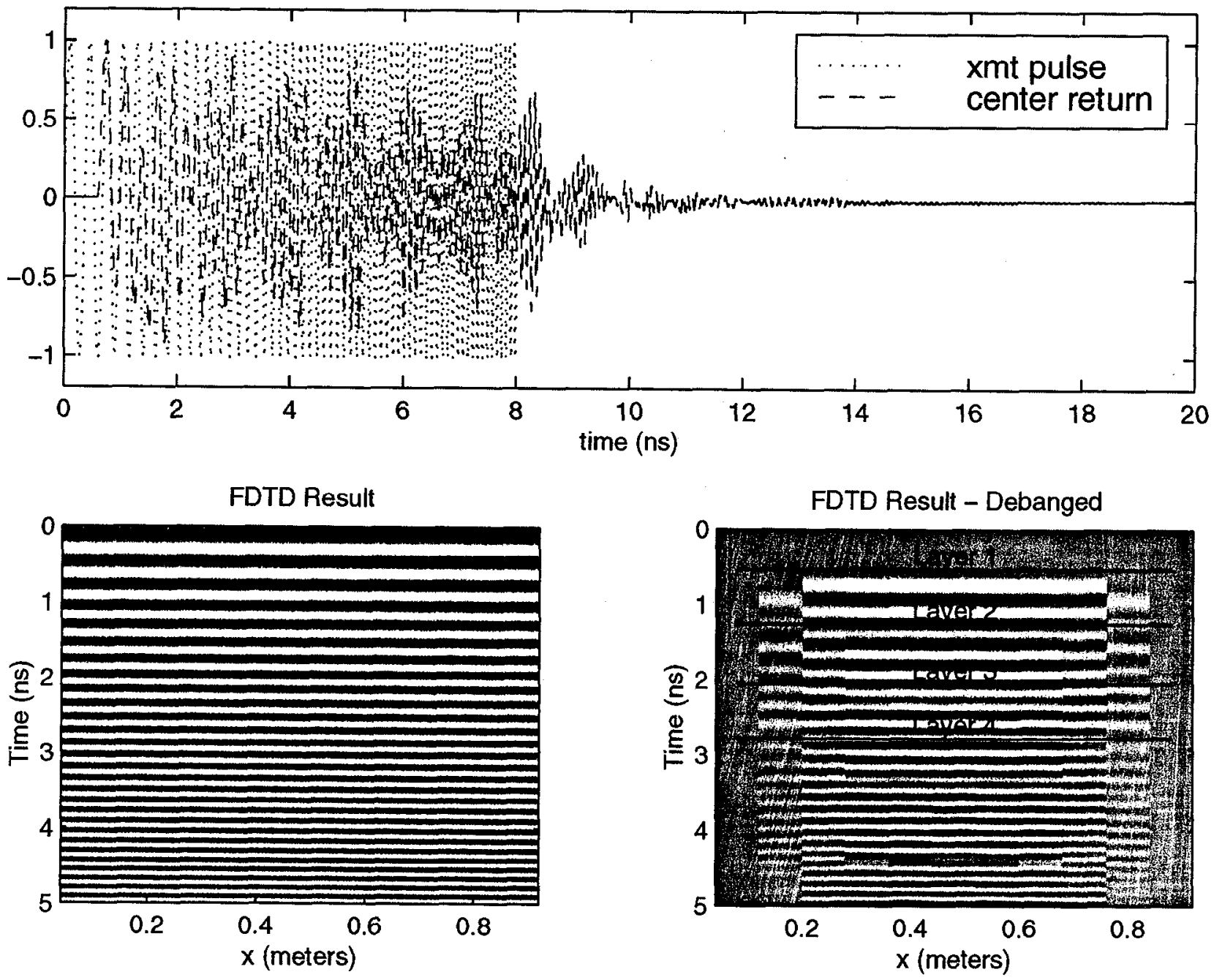

Figure 10: $5 \mathrm{GHz}$ chirp FDTD simulation results. The top plot shows the transmitted pulse and the return extracted from the center of the de-banged result. The left image is the raw simulation output. The right images is the de-banged result, that is, with the large antennato-antenna coupling pulse removed. The de-banged image shows the location of each layer as computed with Eqn. 14. The simulation parameters are listed in Table 4. 
Transmitted $4 \mathrm{~cm} \mathrm{DOG} \mathrm{and} \mathrm{Normalized} \mathrm{Center} \mathrm{Return}$
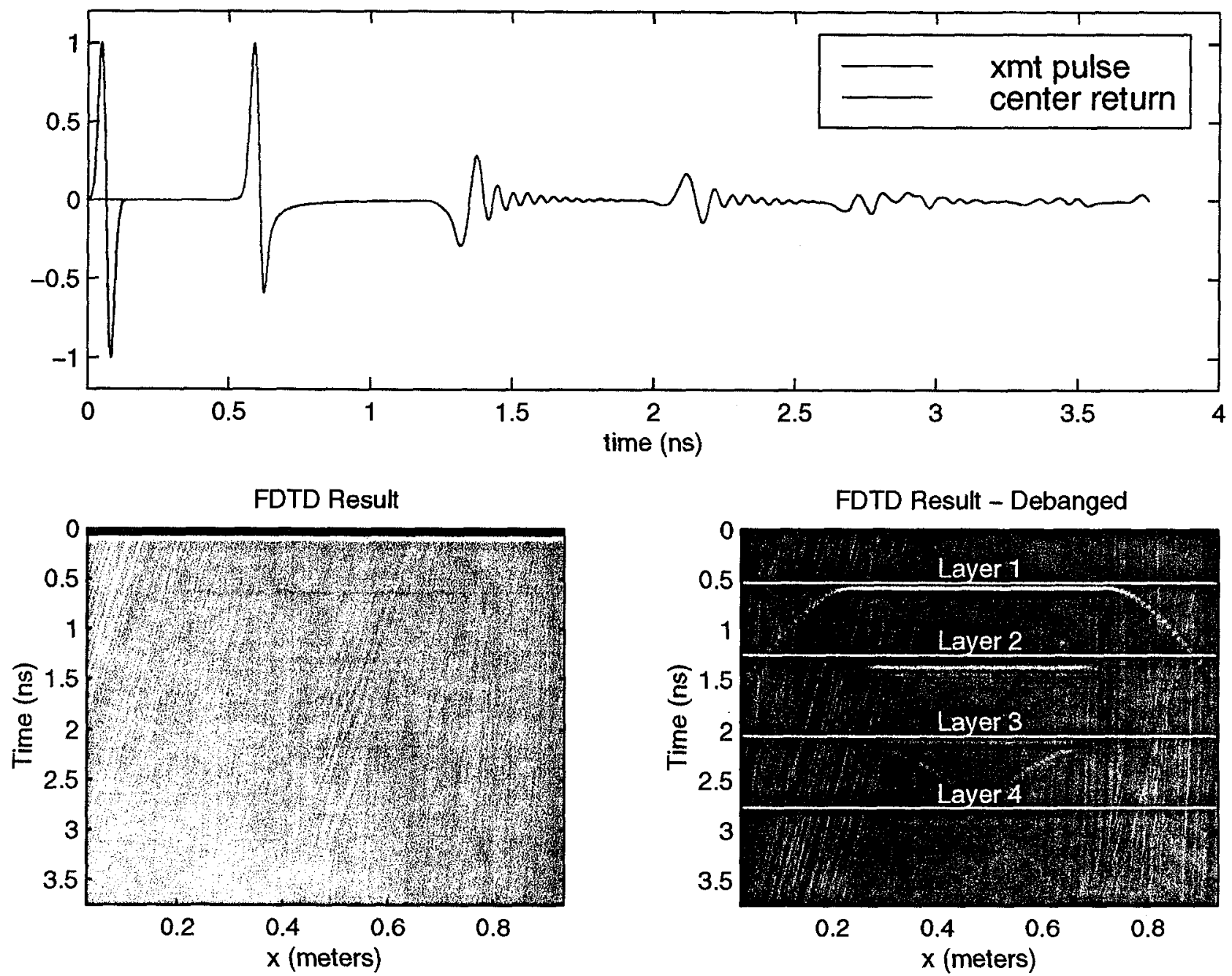

Figure 11: $4 \mathrm{~cm}$ DOG FDTD simulation results. The top plot shows the transmitted pulse and the return extracted from the center of the de-banged result. The left image is the raw simulation output. The right images is the de-banged result, that is, with the large antennato-antenna coupling pulse removed. The de-banged image shows the location of each layer as computed with Eqn. 14. The simulation parameters are listed in Table 4. 


\section{Signal Processing}

The problem of reflection from multiple layers is presented in Figure 12 for the case of four layers. The incident ficld is $A(\omega) e^{i k_{z 0} z}$. At cach layer there will be reflected and transmitted fields as indicated by the amplitude scattering coefficients, $R_{n}, n=0, \ldots$, and $T_{n}, n=1, \ldots$, respectively. These coefficients are determined by the boundary conditions at the interfaces. For an $N$ layer system, the total field is given by

$$
u(z)= \begin{cases}A(\omega) e^{i k_{z 0} z}+R_{0} e^{-i k_{z 0} z} & z \leq L_{1} \\ T_{1} e^{i k_{z 1} z}+R_{1} e^{-i k_{z 1} z} & L_{1}<z \leq L_{2} \\ \vdots & \vdots \\ T_{N-1} e^{i k_{z(N-1)} z}+R_{N-1} e^{-i k_{z(N-1)} z} & L_{N-1}<z \leq L_{N} \\ T_{N} e^{i k_{z N} z} & z>L_{N}\end{cases}
$$

where $k_{z n} \equiv \omega \mu_{0} \epsilon_{n}$ is the $z$ component of the wavenumber within the $n$-th layer. The boundary conditions imposed at the interfaces are [7] continuity of the field and its first derivative. That is, at each layer, we impose

$$
\begin{aligned}
\lim _{z \rightarrow L_{n}^{-}} u(z) & =\lim _{z \rightarrow L_{n}^{+}} u(z), \quad \text { and } \\
\lim _{z \rightarrow L_{n}^{-}} \frac{d}{d z} u(z) & =\lim _{z \rightarrow L_{n}^{+}} \frac{d}{d z} u(z) .
\end{aligned}
$$

This results in $N$ equations for the $N$ unknown coefficients. The general solution for an $N$-layered medium is

$$
\begin{aligned}
& R_{0}=\frac{\left(k_{z 0}-k_{z 1}\right) A(\omega)+2 k_{z 1} R_{1}}{k_{z 0}+k_{z 1}} \\
& R_{n}=\frac{2 k_{z(n+1)} e^{i\left(k_{z n}-k_{z(n+1)}\right) L_{n+1}} R_{n+1}+\left(k_{z n}-k_{z(n+1)}\right) e^{i 2 k_{z n} L_{n+1}} T_{n}}{k_{z n}+k_{z(n+1)}} \\
& T_{1}=\frac{2 k_{z 0} A(\omega)+\left(k_{z 1}-k_{z 0}\right) R_{1}}{k_{z 0}+k_{z 1}} \\
& T_{n}=\frac{e^{-i 2 k_{z n} L_{n}}\left(\left(k_{z n}-k_{z(n-1)}\right) R_{n}+2 k_{z(n-1)} e^{i\left(k_{z(n-1)}+k_{z n}\right) L_{n}} T_{n-1}\right)}{k_{z n}+k_{z(n+1)}} \\
& T_{N}=\frac{2 k_{z(N-1)} e^{i\left(k_{z(N-1)}-k_{z N}\right) L_{N}} T_{N-1}}{k_{z(N-1)}+k_{z N}}
\end{aligned}
$$

where $n$ is the $n$-th layer. This recursive solution has built into it the multiple reflections and transmissions at each layer. Specifically, for the four layer wall, we have

$$
R_{0}=\frac{\left(k_{z 0}-k_{z 1}\right) A(\omega)+2 k_{z 1} R_{1}}{k_{z 0}+k_{z 1}}
$$




$$
\begin{aligned}
& R_{1}=\frac{2 k_{z 2} e^{i\left(k_{z 1}-k_{z 2}\right) L_{2}} R_{2}+\left(k_{z 1}-k_{z 2}\right) e^{i 2 k_{z 1} L_{2}} T_{1}}{k_{z 1}+k_{z 2}} \\
& R_{2}=\frac{2 k_{z 3} e^{i\left(k_{z 2}-k_{z 3}\right) L_{3}} R_{3}+\left(k_{z 2}-k_{z 3}\right) e^{i 2 k_{z 2} L_{3}} T_{2}}{k_{z 2}+k_{z 3}} \\
& R_{3}=\frac{\left(k_{z 3}-k_{z 4}\right) e^{i 2 k_{z 3} L_{4}} T_{3}}{k_{z 3}+k_{z 4}} \\
& T_{1}=\frac{2 k_{z 0} A(\omega)+\left(k_{z 1}-k_{z 0}\right) R_{1}}{k_{z 0}+k_{z 1}} \\
& T_{2}=\frac{e^{-i 2 k_{z 2} L_{2}}\left(\left(k_{z 2}-k_{z 1}\right) R_{2}+2 k_{z 1} e^{i\left(k_{z 1}+k_{z 2}\right) L_{2}} T_{1}\right)}{k_{z 2}+k_{z 3}} \\
& T_{3}=\frac{e^{-i 2 k_{z 3} L_{3}}\left(\left(k_{z 3}-k_{z 2}\right) R_{3}+2 k_{z 2} e^{i\left(k_{z 2}+k_{z 3}\right) L_{3}} T_{2}\right)}{k_{z 2}+k_{z 3}} \\
& T_{4}=\frac{2 k_{z 3} e^{i\left(k_{z 3}-k_{z 4}\right) L_{4}} T_{3}}{k_{z 3}+k_{z 4}} .
\end{aligned}
$$

For the purpose of identifying the layers in a wall, we are not interested in all the multiple transmissions and reflections but rather only the primary reflections as indicated in Figure 13. The figure shows the primary reflections for a four layer medium using the physical parameters of the cinder block wall of Section 2. Only primary reflections are modeled here: at each interface there will be reflected and refracted wave components which we have ignored. At time $t=0$, the pulse is launched. After propagation delays, the primary reflections or echos are received from each layer. $h(\mathbf{r}, t)$ in Eqn. 11 models these delays. Thus, we view the problem as that of a one-dimensional "pulse-ccho" problem in which we launch the pulse, $p(t)$, into a medium, $h(\mathbf{r}, t)$, and wait for the echos, $u(\mathbf{r}, t)$. The time delay between transmission of the original pulse and the reception of the echos determines the layer thickness. We shall show that despite the problems and limitations of the model of Eqn. 11, these issues do not prevent us from determining the layer locations.

Given the one-dimensional convolution model of Eqn. 30 and the expectation of seeing the primary reflections from each of the layers as shown in Figure 3, the problem becomes one of signal processing to perform time delay estimation [16] on the results of the FDTD simulation. With this in mind, we develop an algorithm to locate the layers. We anticipate being able to match the calculated time delays of Table 3 with features in the returns.

As an additional test, we created an ideal, homogeneous environment with point reflectors 


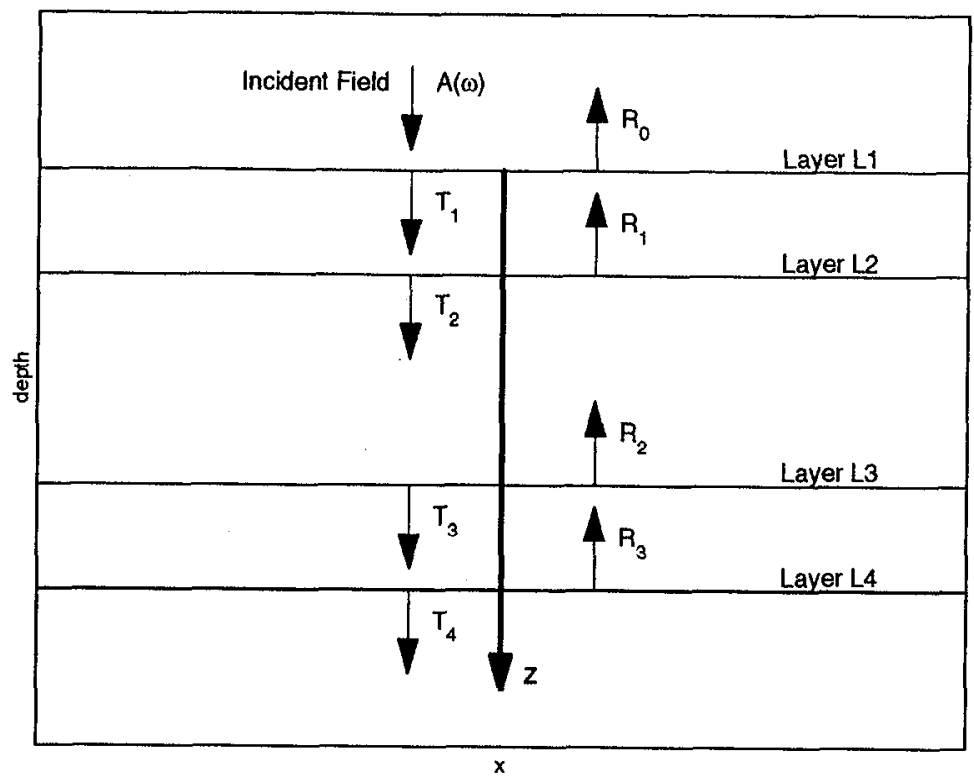

Figure 12: Planar, multi-layer reflection and transmission for the case of four layers. The incident field is $A(\omega) e^{i k_{z 0} z}$. At each interface there will be reflected and transmitted fields.

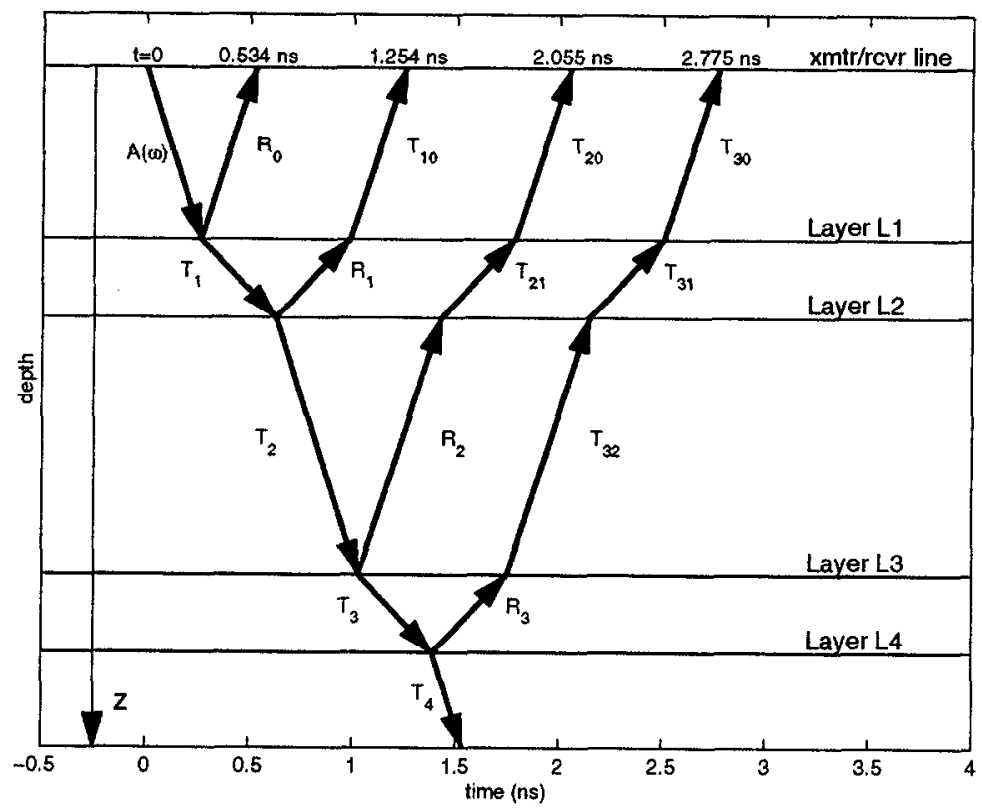

Figure 13: Primary or single reflections from four layers for the simulated cinder block wall of Section 2. The slope of the arrows is the electromagnetic propagation velocity within the layer. The numbers on the transmitter/receiver line are the single reflection arrival times from each layer. 


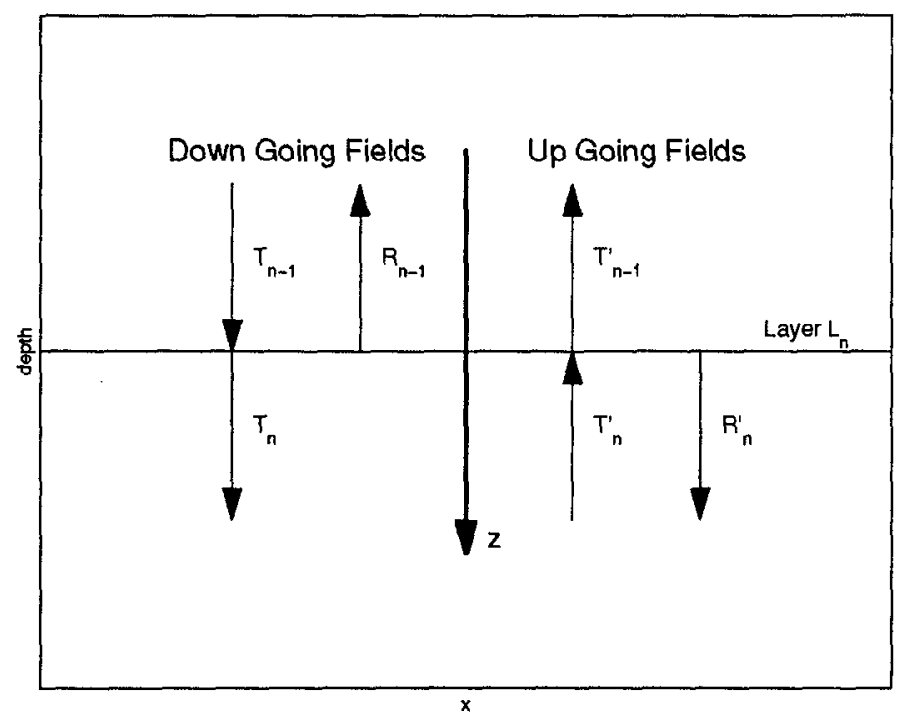

Figure 14: Down and up going transmitted and reflected field coefficients for the $n$-th layer. using the delays from Table 3:

$$
h_{i}(t) \equiv \sum_{n=1}^{4} \alpha_{n} \delta\left(t-\tau_{n}\right),
$$

where the $\alpha_{n}$ are related to the transmission and reflection coefficients for the primary reflections from each layer. To determine the $\alpha_{n}$, consider the fields traveling downward and upward at layer $L_{n}$ :

$$
\begin{aligned}
& u_{\downarrow}(z)= \begin{cases}T_{n-1} e^{i k_{z(n-1)} z}+R_{n-1} e^{-i k_{z(n-1)} z} & L_{n-1}<z \leq L_{n} \\
T_{n} e^{i k_{z n} z} & L_{n}<z \leq L_{n+1}\end{cases} \\
& u_{\uparrow}(z)= \begin{cases}T_{n-1}^{\prime} e^{-i k_{x(n-1)} z} & L_{n-1}<z \leq L_{n} \\
T_{n}^{\prime} e^{-i k_{z n} z}+R_{n}^{\prime} e^{i k_{z n} z} & L_{n}<z \leq L_{n+1}\end{cases}
\end{aligned}
$$

as shown in Figure 14. $u_{\downarrow}(z)$ and $u_{\uparrow}(z)$ represent the total downward and upward traveling fields, respectively, resulting from single reflections at the $n$-th layer. Using the boundary conditions of Eqn. 32, the relationships between the transmission and reflection coefficients for this layer are found to be

$$
\begin{aligned}
R_{n-1} & =T_{n-1} r_{n} e^{i 2 L_{n} k_{z(n-1)}} \\
T_{n} & =T_{n-1}\left(1+r_{n}\right) e^{i L_{n}\left(k_{z(n-1)}-k_{z n}\right)}
\end{aligned}
$$




$$
\begin{aligned}
R_{n}^{\prime} & =-T_{n}^{\prime} r_{n} e^{-i 2 L_{n} k_{z n}} \\
T_{n-1}^{\prime} & =T_{n}^{\prime}\left(1-r_{n}\right) e^{i L_{n}\left(k_{z(n-1)}-k_{z n}\right)}
\end{aligned}
$$

where

$$
r_{n} \equiv \frac{k_{z(n-1)}-k_{z n}}{k_{z(n-1)}+k_{z n}} .
$$

Using these relationships, we can calculate the primary reflection coefficients of Figure 13. For the downward traveling fields, they are:

$$
\begin{aligned}
& T_{1}=A(\omega)\left(1+r_{1}\right) e^{i L_{1}\left(k_{z 0}-k_{z 1}\right)} \\
& T_{2}=T_{1}\left(1+r_{2}\right) e^{i L_{2}\left(k_{z 1}-k_{z 2}\right)} \\
& T_{3}=T_{2}\left(1+r_{3}\right) e^{i L_{3}\left(k_{z 2}-k_{z 3}\right)} \\
& T_{4}=T_{3}\left(1+r_{4}\right) e^{i L_{4}\left(k_{z 3}-k_{z 4}\right)} \\
& R_{0}=A(\omega) r_{1} e^{i L_{1} k_{z 0}} \\
& R_{1}=T_{1} r_{2} e^{i L_{2} k_{z 1}} \\
& R_{2}=T_{2} r_{3} e^{i L_{3} k_{z 2}} \\
& R_{3}=T_{3} r_{4} e^{i L_{4} k_{z 3}} .
\end{aligned}
$$

For the upward traveling fields, we find:

$$
\begin{aligned}
& T_{10}=R_{1}\left(1-r_{1}\right) e^{i L_{1}\left(k_{z 0}-k_{z 1}\right)} \\
& T_{21}=R_{2}\left(1-r_{2}\right) e^{i L_{2}\left(k_{z 1}-k_{z 2}\right)} \\
& T_{32}=R_{3}\left(1-r_{3}\right) e^{i L_{3}\left(k_{z 2}-k_{z 3}\right)} \\
& T_{20}=T_{21}\left(1-r_{1}\right) e^{i L_{1}\left(k_{z 0}-k_{z 1}\right)} \\
& T_{31}=T_{32}\left(1-r_{2}\right) e^{i L_{2}\left(k_{z 1}-k_{z 2}\right)} \\
& T_{30}=T_{31}\left(1-r_{1}\right) e^{i L_{1}\left(k_{z 0}-k_{z 1}\right)} .
\end{aligned}
$$


We may combine Eqns. 41 through 54 to obtain the the primary reflection coefficients at the receiver line of Figure 13:

$$
\begin{aligned}
& R_{0}=A(\omega) r_{1} e^{i L_{1} k_{z 0}} \\
& T_{10}=A(\omega)\left(1-r_{1}^{2}\right) r_{2} e^{i\left(2 L_{1}\left(k_{z 0}-k_{z 1}\right)+L_{2} k_{z 1}\right)} \\
& T_{20}=A(\omega)\left(1-r_{1}^{2}\right)\left(1-r_{2}^{2}\right) r_{3} e^{i\left(2 L_{1}\left(k_{z 0}-k_{z 1}\right)+2 L_{2}\left(k_{z 1}-k_{z 2}\right)+L_{3} k_{z 2}\right)} \\
& T_{30}=A(\omega)\left(1-r_{1}^{2}\right)\left(1-r_{2}^{2}\right)\left(1-r_{3}^{2}\right) r_{4} e^{i\left(2 L_{1}\left(k_{z 0}-k_{z 1}\right)+2 L_{2}\left(k_{z 1}-k_{z 2}\right)+2 L_{3}\left(k_{z 2}-k_{z 3}\right)+L_{4} k_{z 3}\right)}
\end{aligned}
$$

The sum of these coefficients yield the transfer function of the four layer system:

$$
\begin{aligned}
H_{i}(\omega) & =R_{0}+T_{10}+T_{20}+T_{30} \\
& =\sum_{n=1}^{4} \alpha_{n} e^{i \omega \tau_{n}}
\end{aligned}
$$

Transforming back to the time domain we obtain Eqn. 33 where the $\alpha_{n}$ are the amplitudes of the primary reflection coefficients for each layer, that is:

$$
\begin{aligned}
& \alpha_{1} \equiv A(\omega) r_{1} \\
& \alpha_{2} \equiv A(\omega)\left(1-r_{1}^{2}\right) r_{2} \\
& \alpha_{3} \equiv A(\omega)\left(1-r_{1}^{2}\right)\left(1-r_{2}^{2}\right) r_{3} \\
& \alpha_{4} \equiv A(\omega)\left(1-r_{1}^{2}\right)\left(1-r_{2}^{2}\right)\left(1-r_{3}^{2}\right) r_{4}
\end{aligned}
$$

and the $\tau_{n}$ are obtained from the argument of the exponentials.

By comparing the FDTD simulation results with the ideal pulse-echo model of Eqn. 33, we found it was simpler to set $\alpha_{n}=\alpha^{n-1}$ where $\alpha \equiv .5$, rather than computing the coefficients explicitly. Thus Eqn. 33 reduces to

$$
h_{i}(t) \equiv \sum_{n=1}^{4} \alpha^{n-1} \delta\left(t-\tau_{n}\right)
$$

This impulse response is presented in Figure 15 and represents an ideal pulse-echo environment, since the returned waveform is simply attenuated and time-shifted copies of the transmitted pulse. This can be seen by convolving Eqn. 64 with the transmitted pulse, $p(t)$ : 


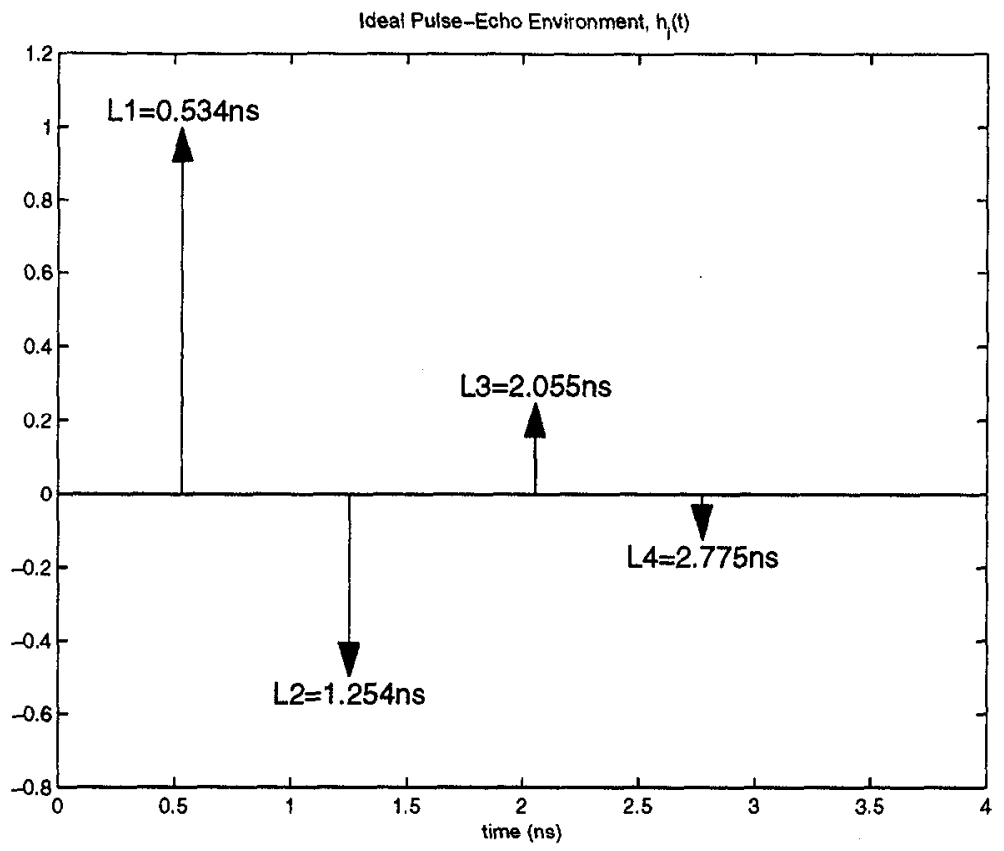

Figure 15: Ideal pulse-echo environment where the impulse response is $h_{i}(t)=$ $\sum_{n=1}^{4} \alpha^{n-1} \delta\left(t-\tau_{n}\right)$. This models the the primary reflection propagation model of Figure 13 . $\alpha=-0.5$.

return using delayed and attenuated copies of the transmitted pulses:

$$
\begin{aligned}
r_{i}(t) & =\int d t^{\prime} h_{i}\left(t-t^{\prime}\right) p\left(t^{\prime}\right) \\
& =\sum_{n=1}^{4} \alpha^{n-1} p\left(t-\tau_{n}\right)
\end{aligned}
$$

We call $r_{i}(t)$ of Eqn. 66 the "ideal pulse-echo return," and in our development and selection of layer detection algorithms, we used both $r(t)$ and $r_{i}(t)$. Note: $r_{i}(t)$ lacks part of the wave propagation physics inherent to $r(t)$ since it models only the primary reflection returns of Figure 13.

There are a number of time delay estimation techniques [16]. After a preliminary investigation, we found the two most successful methods to be those of cross-correlation [16] and deconvolution to estimate the impulse response [17]. These methods require two inputs: the transmitted pulse, $p(t)$, and the returned signal, $r(t)$. As an additional test, we studied a layer detection method which uses only the energy of the returned signal, $|r(t)|^{2}$. We use these methods to generate test signals which are used in the layer detection procedure. 


\subsection{Cross-Correlation}

Given two signals, $p(t)$ and $r(t)$, their cross-correlation is defined as

$$
R_{r p}(t) \equiv\left\langle\int d \iota^{\prime} r\left(\iota^{\prime}\right) p\left(\iota^{\prime}-l\right)\right\rangle
$$

where $\langle\cdot\rangle$ indicates expectation. For deterministic signals, with no noise, Eqn. 67 is simply

$$
R_{r p}(t) \equiv \int d t^{\prime} r\left(t^{\prime}\right) p\left(t^{\prime}-t\right)
$$

We expect to observe peaks in $R_{r p}(t)$ at the shifts were $r(t)$ and $p(t)$ are positively correlated, that is where $r(t)$ contains copies of $p(t)$. To show this, consider $r(t)$ to be a single, translated copy of $p(t)$ :

$$
r(t)=p(t-\tau)
$$

The cross-correlation is then

$$
R_{r p}(t)=\int d t^{\prime} r\left(t^{\prime}\right) p\left(t^{\prime}-t\right)=\int d t^{\prime} p\left(t^{\prime}-\tau\right) p\left(t^{\prime}-t\right)
$$

Evaluate Eqn. 69 at a shift of $\tau$ :

$$
R_{r p}(\tau)=\int d t^{\prime} p\left(t^{\prime}-\tau\right) p\left(t^{\prime}-\tau\right)
$$

and change the integration variable by setting $t^{\prime} \rightarrow t^{\prime}-\tau$,

$$
R_{r p}(\tau)=\int d t^{\prime} p\left(t^{\prime}\right) p\left(t^{\prime}\right)
$$

Eqn 70 is the autocorrelation of $p(t), R_{p p}(t)$, evaluated at zero shift, where

$$
R_{p p}(t) \equiv \int d t^{\prime} p\left(t^{\prime}\right) p\left(t^{\prime}-t\right)
$$

The maximum of the autocorrelation is at the origin [11], thus the maximum of Eqn. 69 occurs at shift $\tau$. Finally, when $r(t)$ contains multiple instances of $p(t), R_{r p}(t)$ will have peaks at each of the shifts. Thus at each layer, that is at each reflection, we expect to see a peak in the cross-correlation where $r(t)$ and $p(t)$ are perfectly correlated (up to an attenuation). 


\begin{tabular}{|l|c|}
\hline Filter Name [16] & Filter Function, $W(\omega)$ \\
\hline Cross-Correlation & $\frac{1}{S_{p p}(\omega)}$ \\
Roth & $\frac{1}{\sqrt{S_{p p}(\omega) S_{r r}(\omega)}}$ \\
PCOT & $\frac{1}{\left|S_{r p}(\omega)\right|}$ \\
Maximum Likelihood & $\frac{\left|\gamma_{r p}(\omega)\right|^{2}}{\left|S_{r p}(\omega)\right|\left[1-\left|\gamma_{r p}(\omega)\right|^{2}\right]}$ where $\gamma_{r p}(\omega) \equiv \frac{S_{r p}(\omega)}{S_{p p}(\omega) S_{r r}(\omega)}$ \\
\hline
\end{tabular}

Table 5: Filter functions used in the cross-correlation time delay estimation techniques.

Techniques exist to enhance the peaks when using cross-correlations to estimate time delays [16]. They operate by filtering the the cross-correlation $R_{r p}(t)$. We summarize the techniques here but defer to [16] for the details. The Fourier transforms of $R_{r p}(t)$ and $R_{p p}(t)$ are defined, respectively, as

$$
\begin{aligned}
S_{r p}(\omega) & =\int d t R_{r p}(t) e^{i \omega t}, \quad \text { and } \\
S_{p p}(\omega) & =\int d t R_{p p}(t) e^{i \omega t},
\end{aligned}
$$

and the filtering of the cross-correlation is expressed as

$$
\hat{R}_{r p}(t)=\frac{1}{2 \pi} \int d \omega W(\omega) S_{r p}(\omega) e^{-i \omega t}
$$

The filtering techniques vary in their choice of the filter function $W(\omega)$. We studied five filter functions including the case when $W(\omega) \equiv 1$. They are listed in Table 5 and we refer to them by the names used in [16]. $\hat{R}_{r p}(t)$ serves as our test signal. Next, we discuss the impulse response estimator. 


\subsection{Impulse Response Estimation}

Impulse response estimation solves the problem presented in Eqn. 13 which we repeat here:

$$
h(\mathbf{r}, \iota)=\mathcal{F}_{\omega}^{-1}\left\{\frac{U(\mathbf{r}, \omega)}{P(\omega)}\right\}
$$

where $\mathcal{F}_{\omega}^{-1}\{\cdot\}$ is the inverse Fourier transform with respect to $\omega$. In solving Eqn. 75 , singularities arise at the zeros of $P(\omega)$. One method to circumvent this is to regularize the fraction by adding a small value to the denominator:

$$
\tilde{h}(\mathbf{r}, t)=\mathcal{F}_{\omega}^{-1}\left\{\frac{U(\mathbf{r}, \omega)}{P(\omega)+\sigma}\right\},
$$

where $\sigma \ll|P(\omega)|$. We found this technique to have very poor performance and we proceeded to use the more robust Weiner solution [17]. Consider the convolution integral of Eqn. 30:

$$
r(t)=\int d t^{\prime} h\left(x_{0}, z_{0}, t-t^{\prime}\right) p\left(t^{\prime}\right)
$$

To develop the Weiner solution, we first Fourier transform Eqn. 77,

$$
R(\omega)=H\left(x_{0}, z_{0}, \omega\right) P(\omega)
$$

Multiply Eqn. 78 by the complex conjugate of $P(\omega)$ and take the expectation value of the result to obtain

$$
\begin{aligned}
\left\langle R(\omega) P^{*}(\omega)\right\rangle & =\left\langle H\left(x_{0}, z_{0}, \omega\right)|P(\omega)|^{2}\right\rangle \\
& =H\left(x_{0}, z_{0}, \omega\right)\left\langle|P(\omega)|^{2}\right\rangle
\end{aligned}
$$

where $H\left(x_{0}, z_{0}, \omega\right)$ is moved out of the expectation operator since it is deterministic. We recognize $\left\langle R(\omega) P^{*}(\omega)\right\rangle$ and $\left\langle|P(\omega)|^{2}\right\rangle$ as being the spectral representations of the crosscorrelation and autocorrelation, respectively. Thus we express Eqn. 79 as

$$
S_{r p}(\omega)=H\left(x_{0}, z_{0}, \omega\right) S_{p p}(\omega)
$$

Solving for the transfer function, we find

$$
\tilde{H}\left(x_{0}, z_{0}, \omega\right)=\frac{S_{r p}(\omega)}{S_{p p}(\omega)+\sigma}
$$


where $\sigma$ is a regularization parameter. Inverse Fourier transforming yields the desired estimate of the impulse response

$$
\tilde{h}\left(x_{0}, z_{0}, t\right)=\mathcal{F}_{\omega}^{-1}\left\{\frac{S_{r p}(\omega)}{S_{p p}(\omega)+\sigma}\right\},
$$

which serves as our test signal. Through trial and error, we chose $\sigma=10^{-6}$ as the regularization parameter.

\subsection{Energy Method}

The energy method requires only the returned signal, $r(t)$. We compute the square of its magnitude, $|r(t)|^{2}$, and search for peaks within this signal without attempting to enhance them.

Given the cross-correlation, $\hat{R}_{r p}(t)$, estimate of the impulse response, $\tilde{h}\left(x_{0}, z_{0}, t\right)$, and the energy signal, $|r(t)|^{2}$, as test signals, we now concentrate on determining the temporal locations of the peaks. Within the model of Eqn. 11, we expect the peaks to coincide with individual layers.

\subsection{Detection Method}

Before discussing the detection method, we digress briefly to cover the discretization of continuous signals for processing on digital computers. Let $s(t)$ be a signal which is a function of the continuous time variable $t$. We call $s(n)$ the sampled version of $s(t)$ and define it as

$$
s(n) \equiv s\left(t_{0}+n \Delta t\right), \quad n=0, \ldots, N,
$$

where $t_{0}$ is the time origin and $\Delta t$ is the sample interval. $\Delta t$ cannot be chosen arbitrarily but must be selected to satisfy the Nyquist sampling theorem [15] which states the sample rate, $1 / \Delta t$, must at least twice the highest frequency content of the signal to be sampled as in Eqn. 25.

The detection method locates peaks in the sampled test signals $\hat{R}_{r p}(n), \tilde{h}\left(x_{0}, z_{0}, n\right)$, or $|r(t)|^{2}$. It is assumed these peaks result from reflections at the layers. The detection procedure involves differencing the test signal and searching for sign changes. Since differencing 
emphasizes noise, we smooth the signal first. Let $s(n)$ be the test signal. The smoothed signal is

$$
\bar{s}(n)=\sum_{m}^{N} s(m) w(n-m)
$$

where $w(n)$ is a rectangular window defined by

$$
w(n)= \begin{cases}1 / A_{\text {win }} & 0 \leq n<A_{\text {win }} \\ 0 & \text { otherwise }\end{cases}
$$

We then difference $\bar{s}(n)$ and look for sign changes which occur at peaks above a specified threshold, $T$. The differenced signal is

$$
\Delta \bar{s}(n) \equiv \bar{s}(n+1)-\bar{s}(n)
$$

Thus a peak is detected at $\bar{s}(n)$ if $\Delta \bar{s}(n)>0$ and $\Delta \bar{s}(n+1)<0$ and $\bar{s}(n)>T$. The peak detection procedure is demonstrated in Figure 16.

As test signals, we used the five filtered cross-correlations, $\hat{R}_{r p}(t)$, from Section 3.1, the

estimated impulse response, $\tilde{h}\left(x_{0}, z_{0}, n\right)$, from Section 3.2, and the energy signal, $|r(t)|^{2}$, from Section 3.3. We applied these methods to the FDTD returns, $r(t)$, of Eqn. 30, and the ideal pulse-echo returns of Eqn. 66 for each one of the five test pulses. The results are presented in the next section.

\section{Results}

To facilitate comparison of detection results, we chose the averaging window size, $A_{\text {win }}$, to be 25 points for all methods. The threshold was set to 0.2 for the cross-correlation, impulse response and energy methods; and 0.15 for maximum likelihood, Roth, PHAT, and SCOT since their signal magnitudes were much less than the impulse response, cross-correlation, and energy methods. The disadvantage of using a common window/threshold parameter pair is that a parameter set which is favorable for one method may be unfavorable for another. In selecting the parameters, we tried to maximize the correct detections while minimizing the false detections. With these constraints in mind, we settled upon the values listed above. 


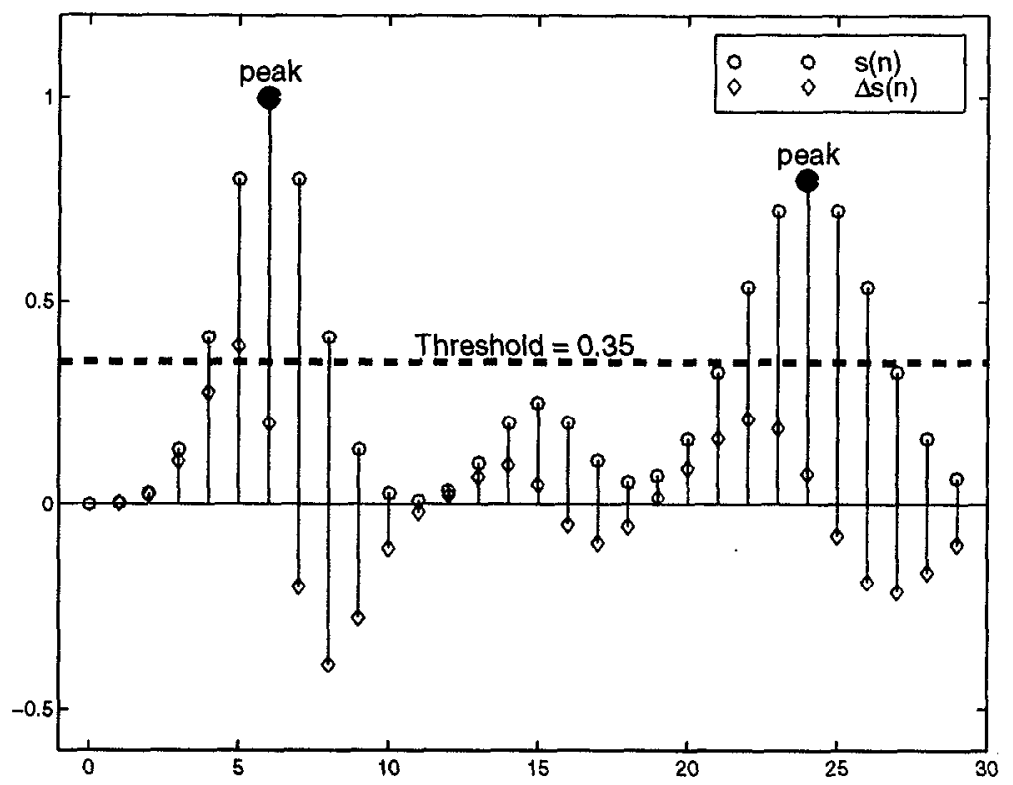

Figure 16: Demonstration of peak detection procedure. The signal, $s(n)$, is differenced to create $\Delta s(n)$. Sign changes within $\Delta s(n)$ which occur at values of $s(n)$ above the threshold of 0.35 are considered to be detected peaks within $s(n)$.

The detection results are tabulated in Tables 6 through 10 as percentage error in layer detection. If $\tau_{n}$ is the calculated time from Table 3 , and $\tau_{n}^{\prime}$ the estimated time from a peak detection in the test signal, the error in layer detection is

$$
e=100 \times \frac{\left|\tau_{n}-\tau_{n}^{\prime}\right|}{\tau_{n}}
$$

In many cases there were multiple false detections. For these cases, we chose the peak closest to the true value in computing the error. The maximum likelihood, Roth, PHAT, and SCOT methods were more prone to multiple false detections than the impulse response and cross-correlation methods. All methods performed better in the ideal cases with their simple kernels. The fourth layer was rarely detected.

In selecting a method to locate layers, we used the criteria of low detection error with minimal false detections. With these conditions, we down-selected from seven to two methods: impulse response and cross-correlation. The graphical results for these two cases are presented in Figures 18 through 21. As a reference we also include the results for the energy method in Figures 22 and 23. The figures show the smoothed test signal, $\bar{s}(n)$, the threshold, $T$, the detected peaks indicating the primary reflection from a layer, and the computed 


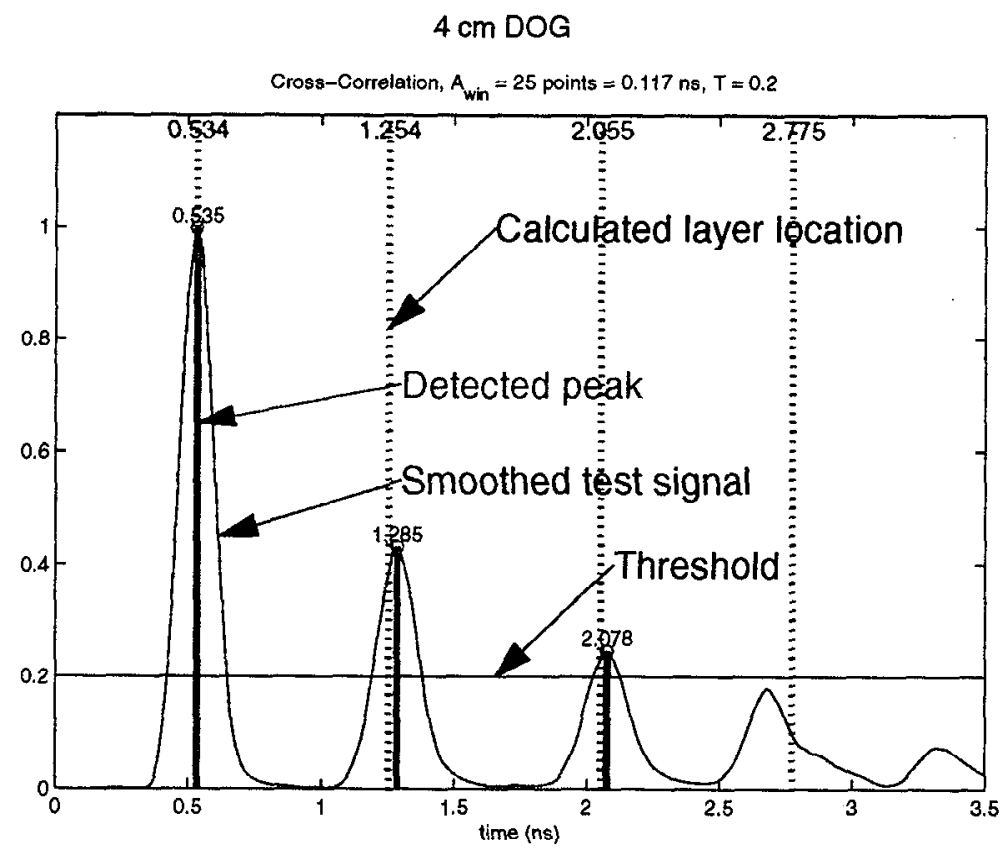

Figure 17: Annotated key to reading the results presented in Figures 18 through 23.

primary reflection arrival time. A key to reading the result figures is shown in Figure 17.

The results lead us to believe the current MIR pulses are not optimal for identifying the layers of a cinder block wall. There are two principal limitations: the long ringing and insufficient bandwidth. The former results in increased reverberation within the wall which masks the primary reflection returns from the layers. As is demonstrated in the $4 \mathrm{~cm} \mathrm{DOG}$ results, a temporally shorter pulse with wider spectral bandwidth permits us to discern more easily the layers using the simple cross-correlation method. Future MIR hardware modifications will result in shorter pulses with wider bandwidths which will improve layer detectability.

\section{Application to Real Data}

We collected data from four test cinder block walls. Each wall was four blocks high and two to three blocks wide. We labeled the walls 1 through 4 . The voids in the cinder blocks of each wall were filled with a different material as follows:

1. Empty, that is the voids were air; 


\section{Pea gravel;}

\section{Cement;}

4. Cement with rebar down the middle.

We collected data from four antenna positions, labeled 'a' through 'd', as shown in Figure 24. The antenna was offset from the front surface of the wall by $9.5 \mathrm{~cm}$. At each position, 100 measurements were taken and averaged to reduce noise. A background measurement was taken out of doors by pointing the antenna up into open space and collecting 100 returns which were then averaged to reduce noise. This mean background measurement was subtracted from the mean wall returns before processing in order to reduce system noise. A measurement of the pulse was collected by capturing the return off of a metal plate. This served as the input, $p(t)$, in the signal processing algorithms.

The results are presented in Figures 25 through 26. For all cases, we used an averaging window size of 25 with a threshold of 0.2 . Each plot is annotated with a vertical line indicating the expected primary return from the front surface of the wall. Since this line does not consistently align with the first peak, we conclude the radar data time origin and sample interval were not accurately calibrated. The overall results are poor, however the plots show the cross-correlation method out-performed the other two methods. The ability to locate the wall layers is highly dependent upon the position of the radar as well as the content of the voids. The detection performance drops when the voids are filled with material which results in increased scattering and returned signal loss. Layer 2 is almost always detected, if not accurately.

\section{Conclusions}

The goal of the OST Wall Surveyor is to locate the layers of a wall probed via a monostatic MIR antenna. Our approach in solving the problem was to perform FDTD simulations, develop and down-select working algorithms based upon the simulation results. We studied two classes of signal processing algorithms: impulse response estimation and correlation. 
Within the latter, we studied studied the five types of filtered cross-correlation listed in Table 5. As an additional test, studied the possibility of locating the layers by detecting peaks in the energy of the returned signal. We applied our algorithms to the FDTD results as well as to an ideal pulse-echo signal to determine performance. As success criteria, we used low detection error with minimal multiple false detection. Comparing the results using these criteria, we down-selected two detection procedures: impulse response and conventional cross-correlation. The methods have two free parameters which must be selected by the user: an averaging window size to reduce noise, and a detection threshold.

We collected data sets from four cinder block test walls. Each wall was filled with a different material. For each wall, we collected data from four separate locations. We used the impulse response, cross-correlation, and energy methods for detecting the layers.

The results lead us to believe the current MIR pulses are not optimal for solving such a problem, however the simulated results show that a shorter pulse with wider bandwidth could be used to detect the layers. Current MIR hardware development is toward that goal.

\section{Acknowledgments}

I am grateful to Dr. Jeffrey Mast for his help and patience with the radar hardware and software, as well as with the FDTD code usage. I wish to thank Dr. James Candy for his technical advice, and Dr. Stephen Azevedo for his support. Additionally, I want to thank Ming Liu, Pat Welsh, and Greg Dallum for their invaluable radar hardware and software help. 
Range Finder

\begin{tabular}{|c|c|c|c|c|}
\hline Method & Layer 1 & Layer 2 & Layer 3 & Layer 4 \\
\hline Impulse Response & $5 \%$ & $3 \%$ & $4 \%$ & $15 \%$ \\
\hline Cross-Correlation & $4 \%$ & $0 \%$ & - & - \\
\hline Max. Like.* & $2 \%$ & $1 \%$ & $9 \%$ & $4 \%$ \\
\hline $\operatorname{Roth}^{*}$ & $3 \%$ & $1 \%$ & $6 \%$ & $10 \%$ \\
\hline PHAT $^{*}$ & $5 \%$ & $1 \%$ & $4 \%$ & $9 \%$ \\
\hline SCOT & $4 \%$ & $1 \%$ & $5 \%$ & $9 \%$ \\
\hline Energy & $5 \%$ & $13 \%$ & - & - \\
\hline
\end{tabular}

Ideal Range Finder

\begin{tabular}{|c|c|c|c|c|}
\hline Method & Layer 1 & Layer 2 & Layer 3 & Layer 4 \\
\hline Impulse Response & $1 \%$ & $0 \%$ & $0 \%$ & - \\
\hline Cross-Correlation & $1 \%$ & $1 \%$ & - & $4 \%$ \\
\hline Max. Like.* & $1 \%$ & $0 \%$ & $4 \%$ & $3 \%$ \\
\hline Roth* & $1 \%$ & $2 \%$ & $2 \%$ & $4 \%$ \\
\hline PHAT & $1 \%$ & $0 \%$ & $0 \%$ & - \\
\hline SCOT & $1 \%$ & $0 \%$ & - & - \\
\hline Energy & $4 \%$ & $7 \%$ & - & - \\
\hline
\end{tabular}

Table 6: Percentage error in layer detection. "** indicates there were multiple false detections. "-" indicates the layer was not detected. 
High Speed Radar

\begin{tabular}{|c|c|c|c|c|}
\hline Method & Layer 1 & Layer 2 & Layer 3 & Layer 4 \\
\hline Impulse Response & $6 \%$ & - & - & - \\
\hline Cross-Correlation & $2 \%$ & $5 \%$ & $21 \%$ & - \\
\hline Max. Like. & $2 \%$ & $11 \%$ & $6 \%$ & $3 \%$ \\
\hline Roth & - & - & - & - \\
\hline PHAT* & $5 \%$ & $9 \%$ & $7 \%$ & $12 \%$ \\
\hline $\mathrm{SCOT}^{*}$ & $5 \%$ & $9 \%$ & $2 \%$ & $12 \%$ \\
\hline Energy & $2 \%$ & - & - & - \\
\hline
\end{tabular}

Ideal High Speed Radar

\begin{tabular}{|l|llll|}
\hline Method & Layer 1 & Layer 2 & Layer 3 & Layer 4 \\
\hline Impulse Response & $1 \%$ & $0 \%$ & $0 \%$ & - \\
Cross-Correlation & $1 \%$ & $0 \%$ & $1 \%$ & $4 \%$ \\
Max. Like.* & $1 \%$ & $0 \%$ & $4 \%$ & $3 \%$ \\
Roth & $1 \%$ & $2 \%$ & $13 \%$ & $9 \%$ \\
PHAT & $1 \%$ & $0 \%$ & $13 \%$ & - \\
SCOT & $1 \%$ & $0 \%$ & $0 \%$ & - \\
Energy & $5 \%$ & - & - & - \\
\hline
\end{tabular}

Table 7: Percentage error in layer detection. "*” indicates there were multiple false detections. "-" indicates the layer was not detected. 


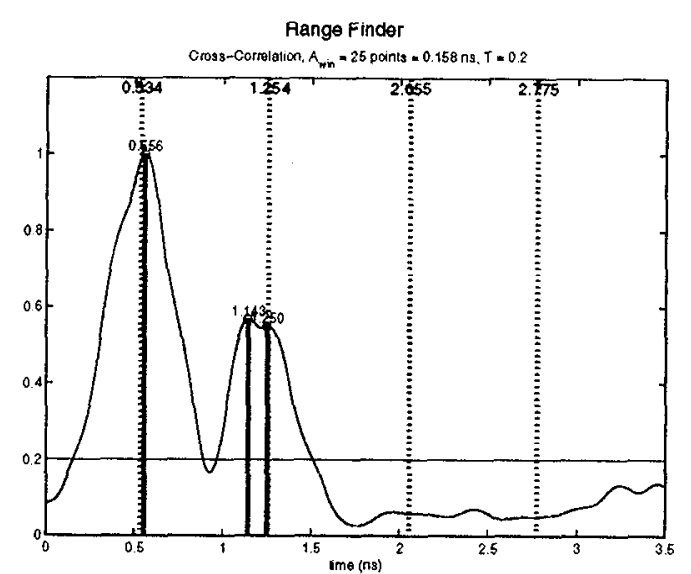

(a)

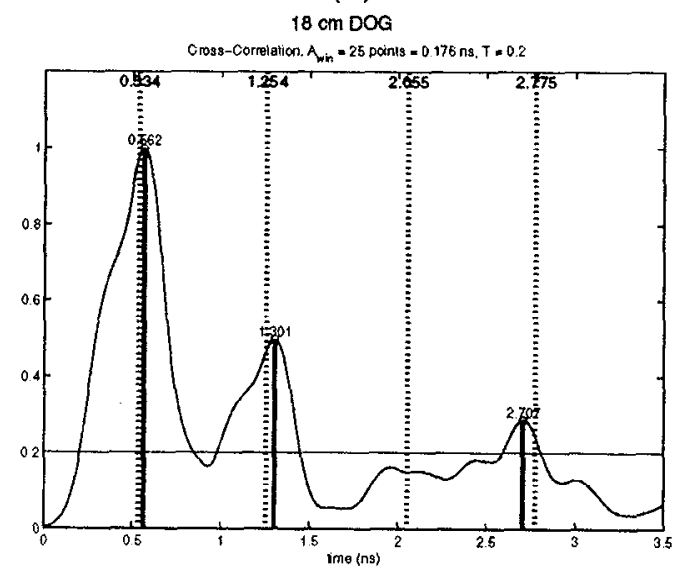

(c)

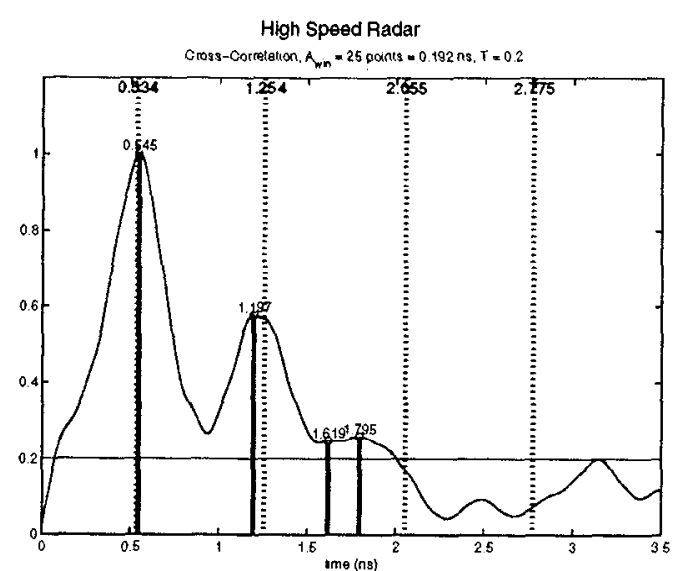

(b)

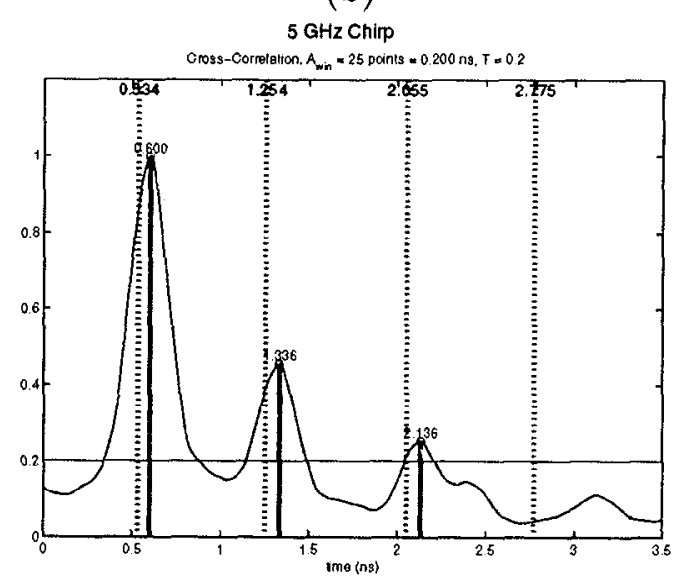

(d)

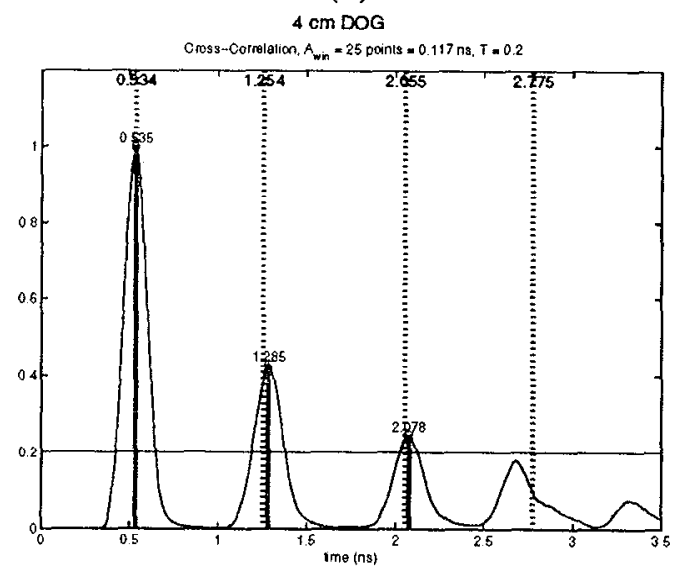

(e)

Figure 18: Cross-correlation detection method for the five pulses. The true primary reflection arrival times as listed in Table 3 are indicated by the dashed lines with the times across the top. The horizontal line is the threshold. 


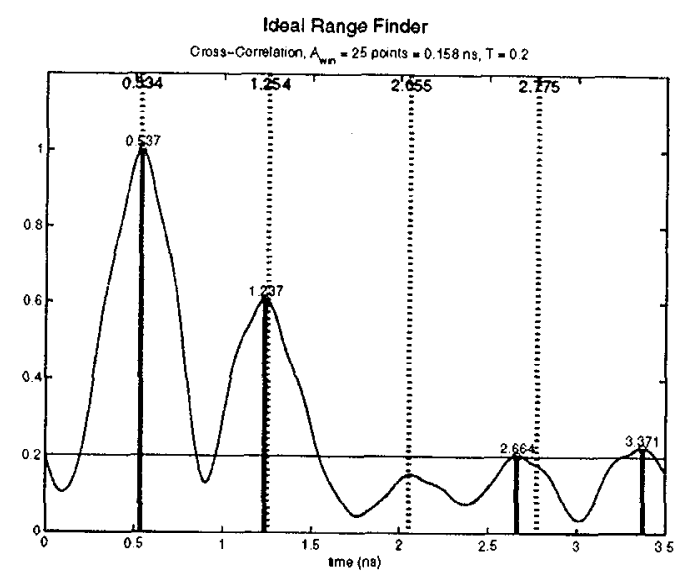

(a)

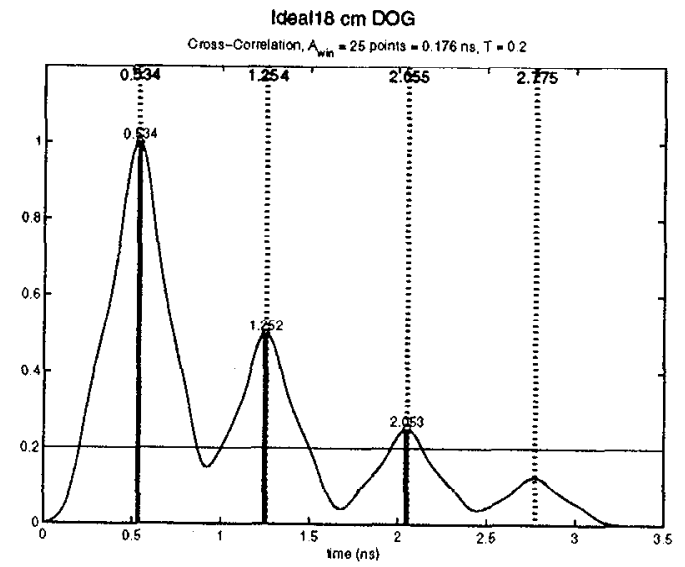

(c)

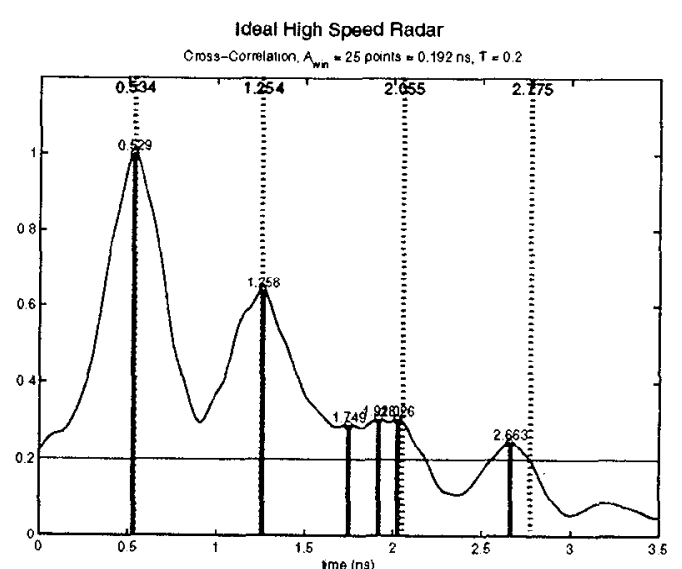

(b)

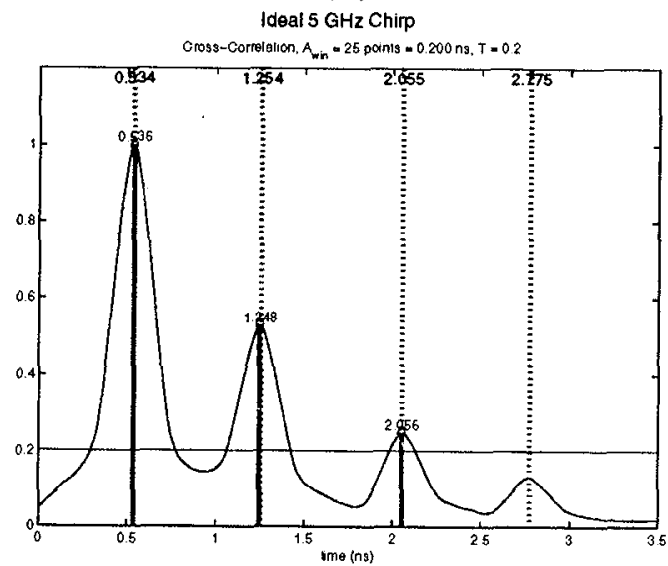

(d)

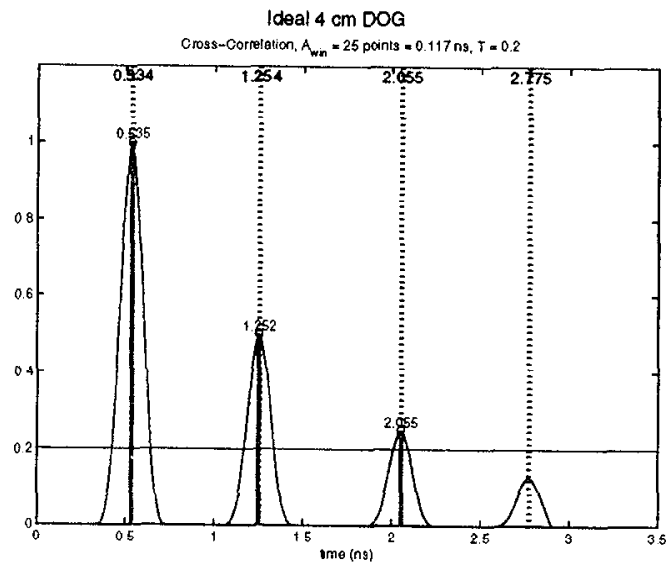

(e)

Figure 19: Cross-correlation detection method for the five ideal cases. The true primary reflection arrival times as listed in Table 3 are indicated by the dashed lines with the times across the top. The horizontal line is the threshold. 


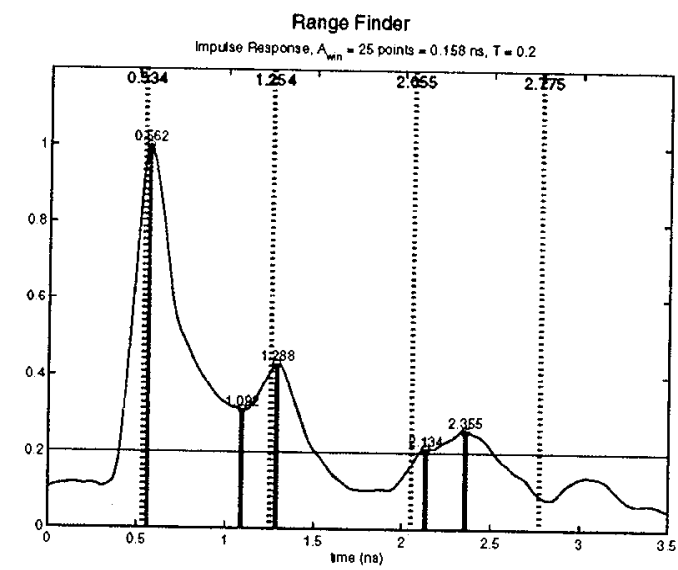

(a)

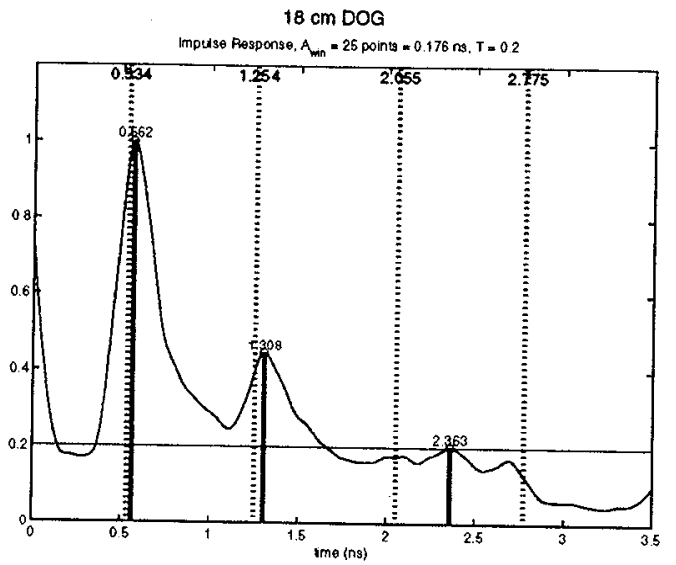

(c)

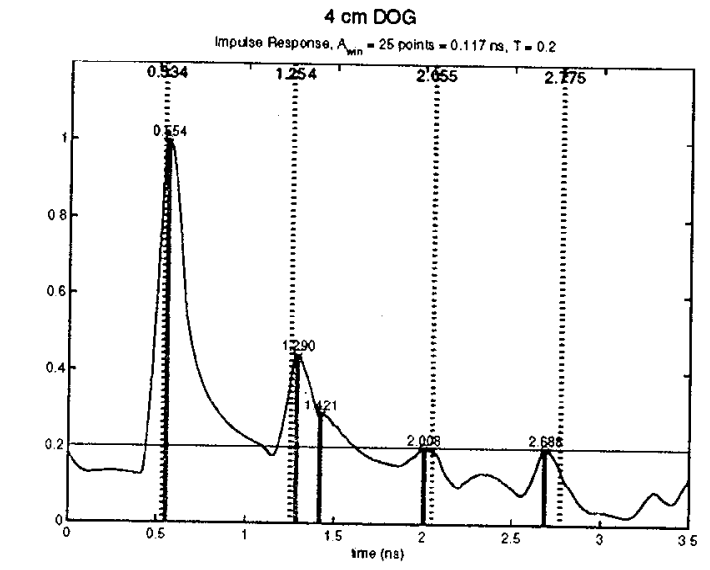

(e)

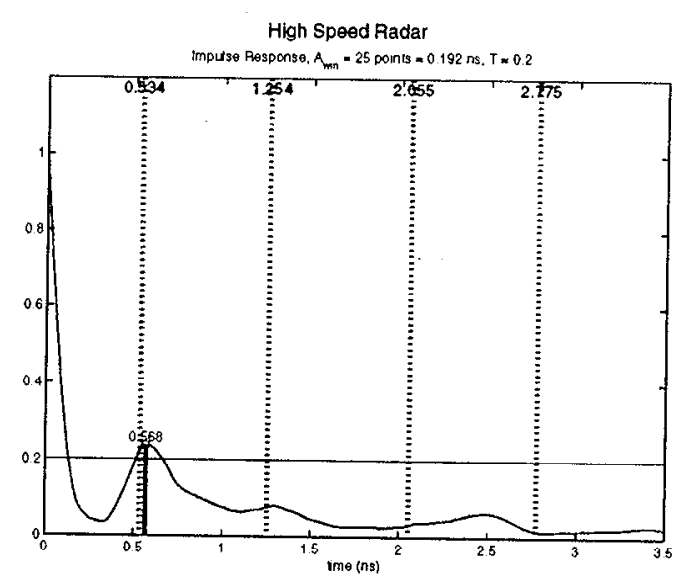

(b)

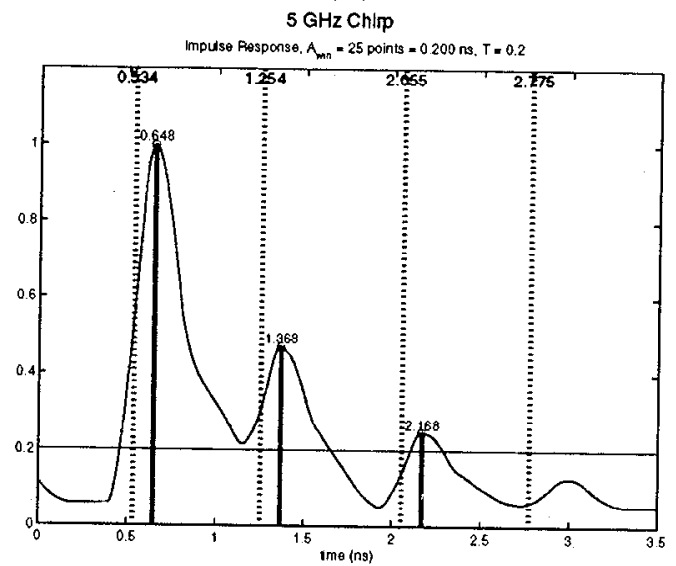

(d)

Figure 20: Impulse response detection method for the five pulses. The true primary reflection arrival times as listed in Table 3 are indicated by the dashed lines with the times across the top. The horizontal line is the threshold. 


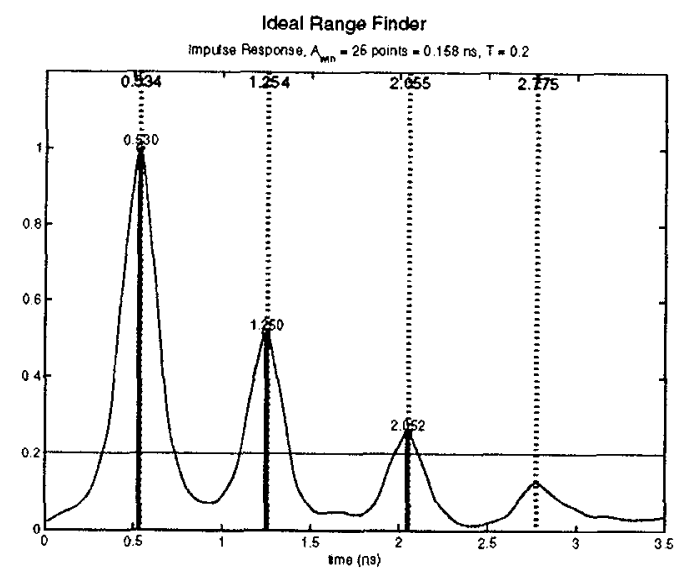

(a)

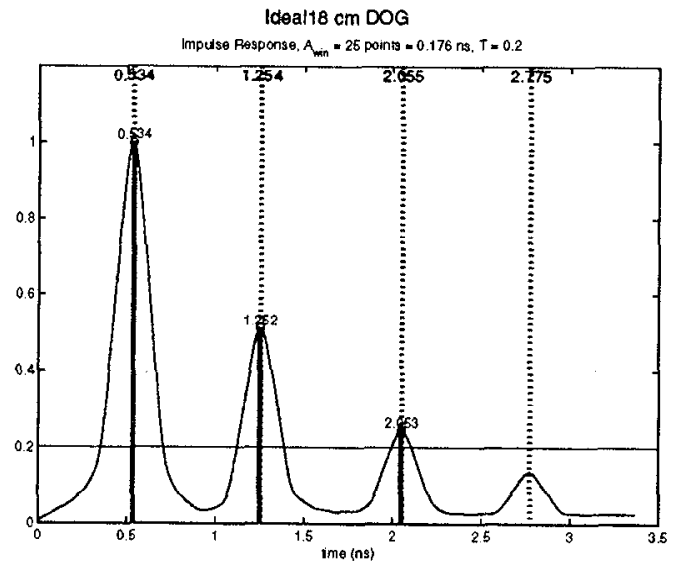

(c)

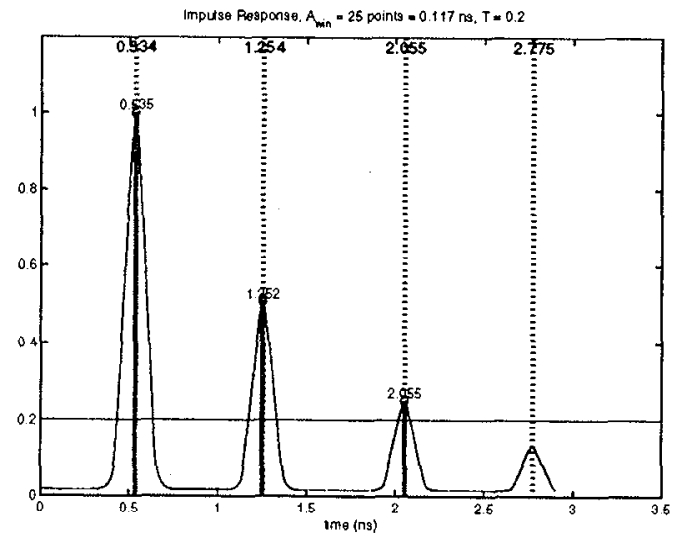

(e)

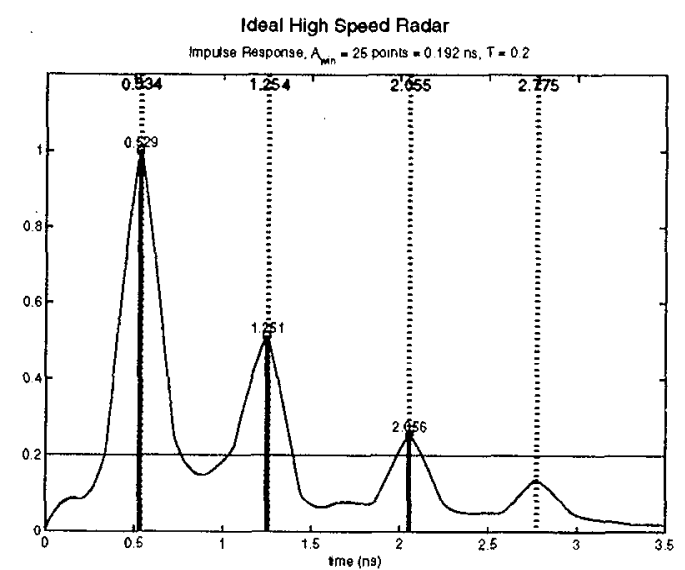

(b)

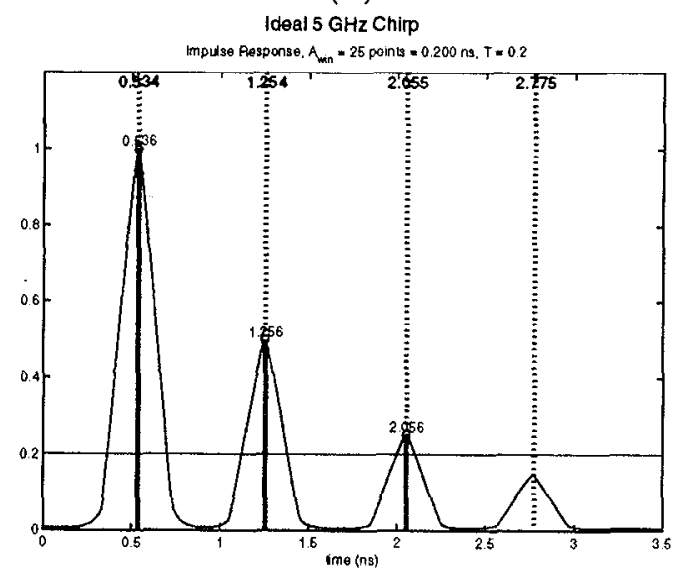

(d)

Figure 21: Impulse response detection method for the five ideal cases. The true primary reflection arrival times as listed in Table 3 are indicated by the dashed lines with the times across the top. The horizontal line is the threshold. 


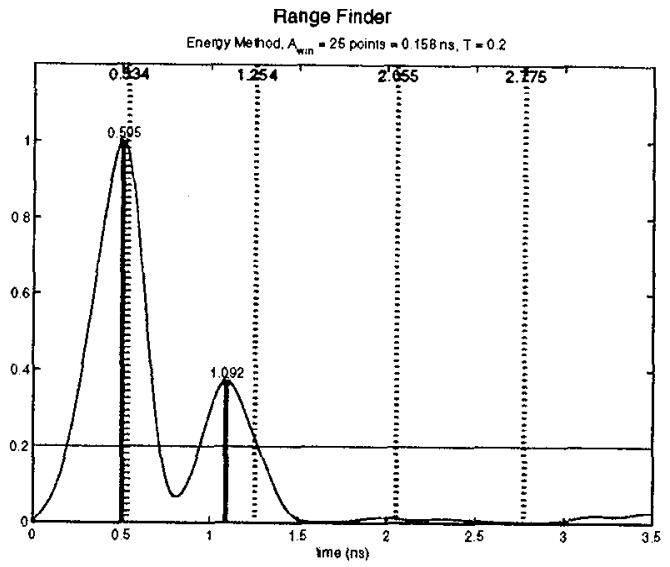

(a)

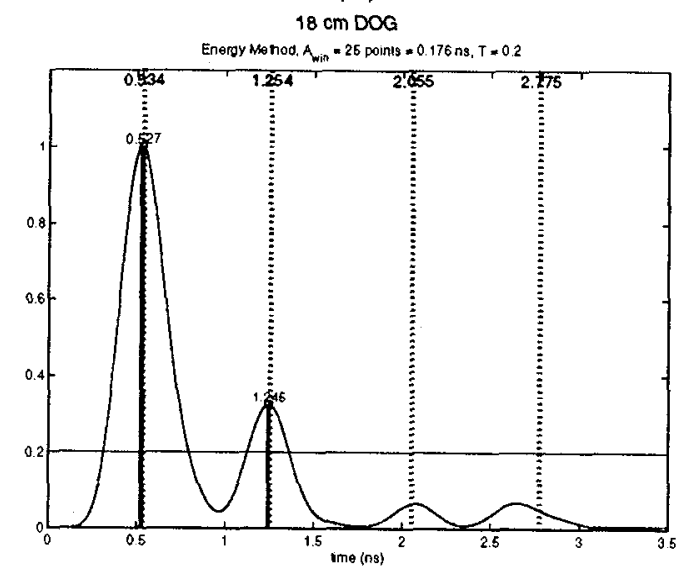

(c)

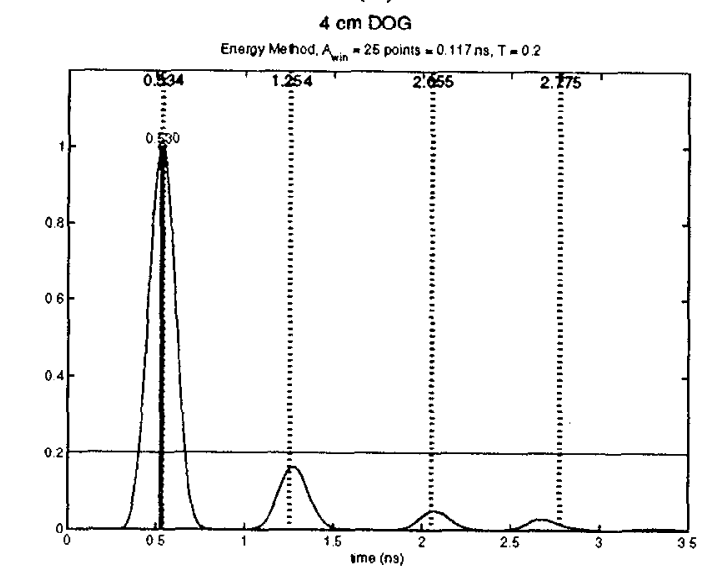

(e)

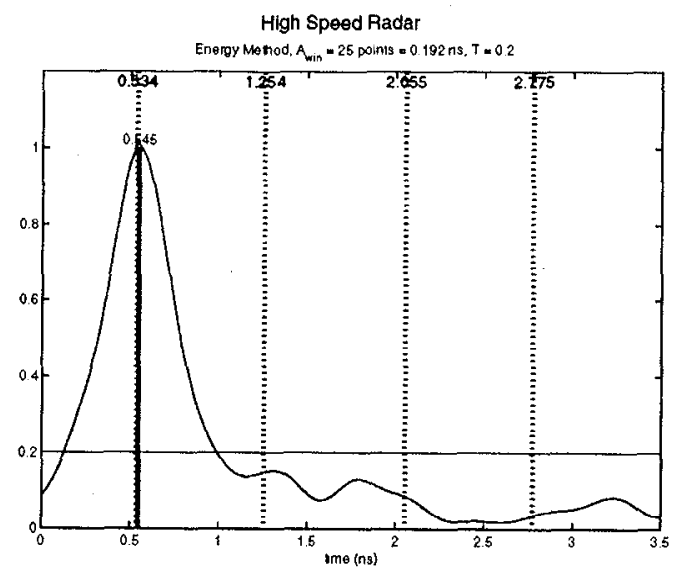

(b)

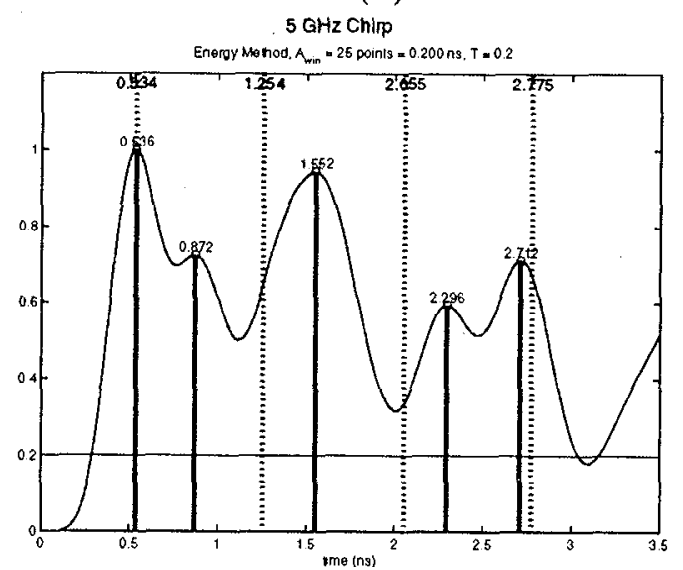

(d)

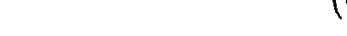




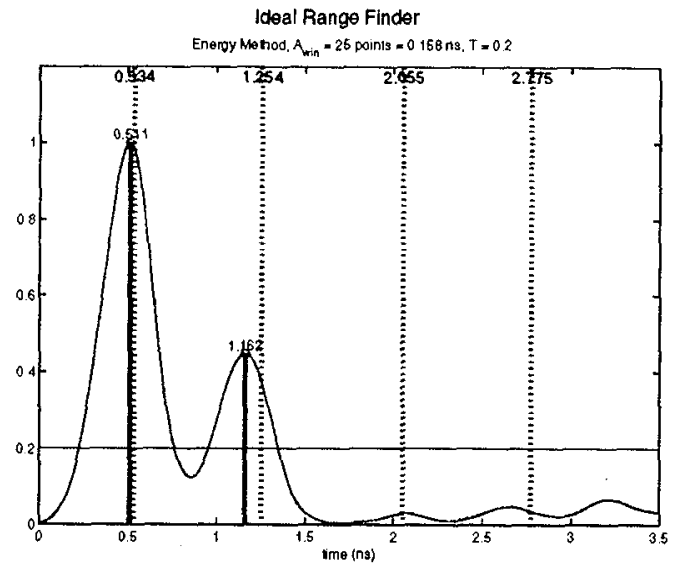

(a)

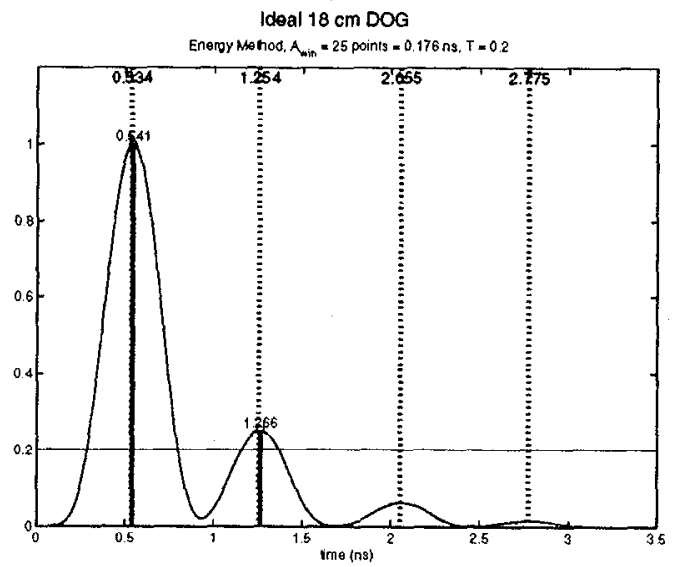

(c)

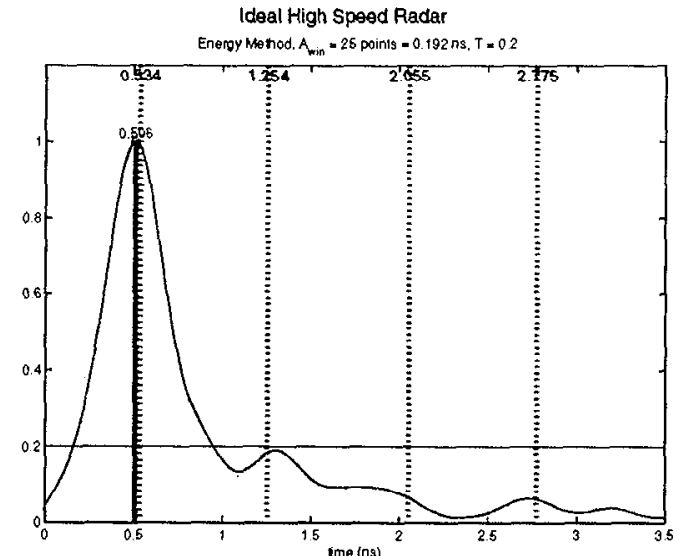

(b)

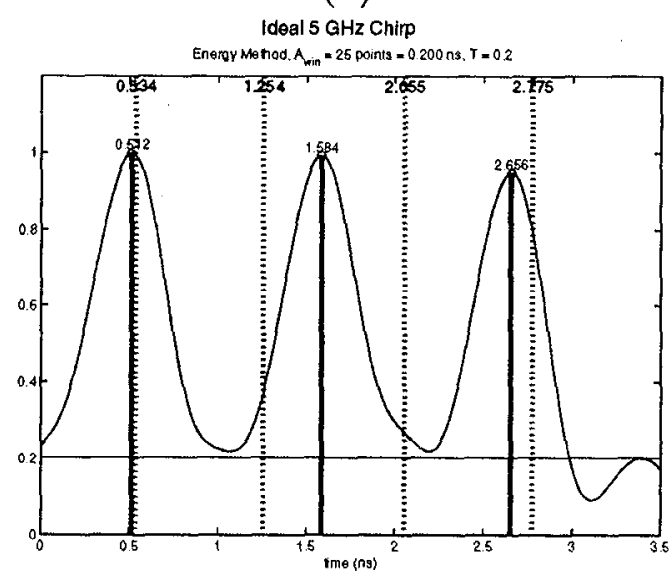

(d)

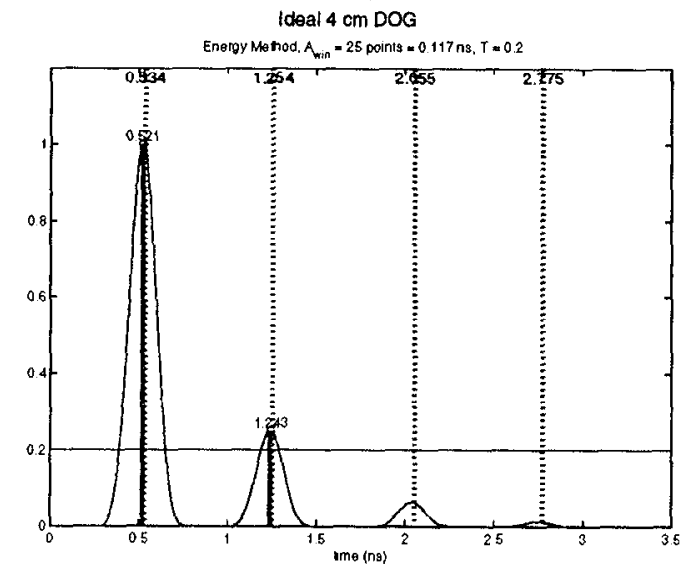

(e)

Figure 23: Energy detection method for the five ideal cases. The true primary reflection arrival times as listed in Table 3 are indicated by the dashed lines with the times across the top. The horizontal line is the threshold. 


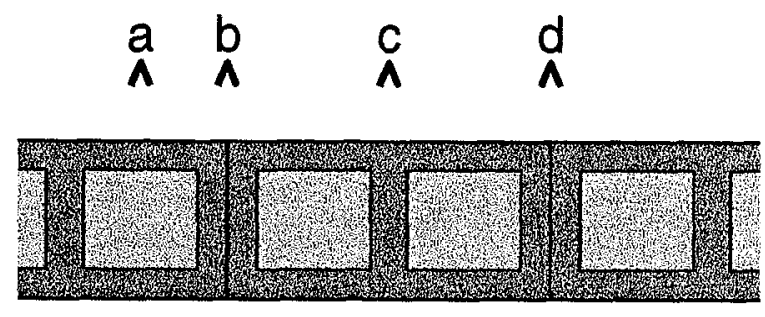

TOP VIEW

FRONT VIEW

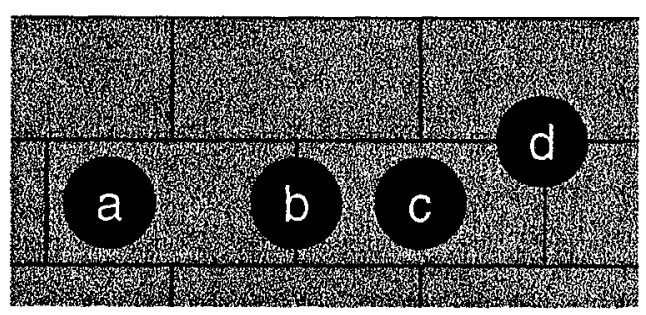

Figure 24: Four antenna positions where data were collected from each of the four test walls. Location ' $a$ ' was in front of a void in the cinder block. Location ' $b$ ' was at the abutting ends of two blocks. Location 'c' was in front of the divider between the block's two voids. Location 'd' was in front of the interface of three blocks. The antenna was $9.5 \mathrm{~cm}$ off of the front surface. 

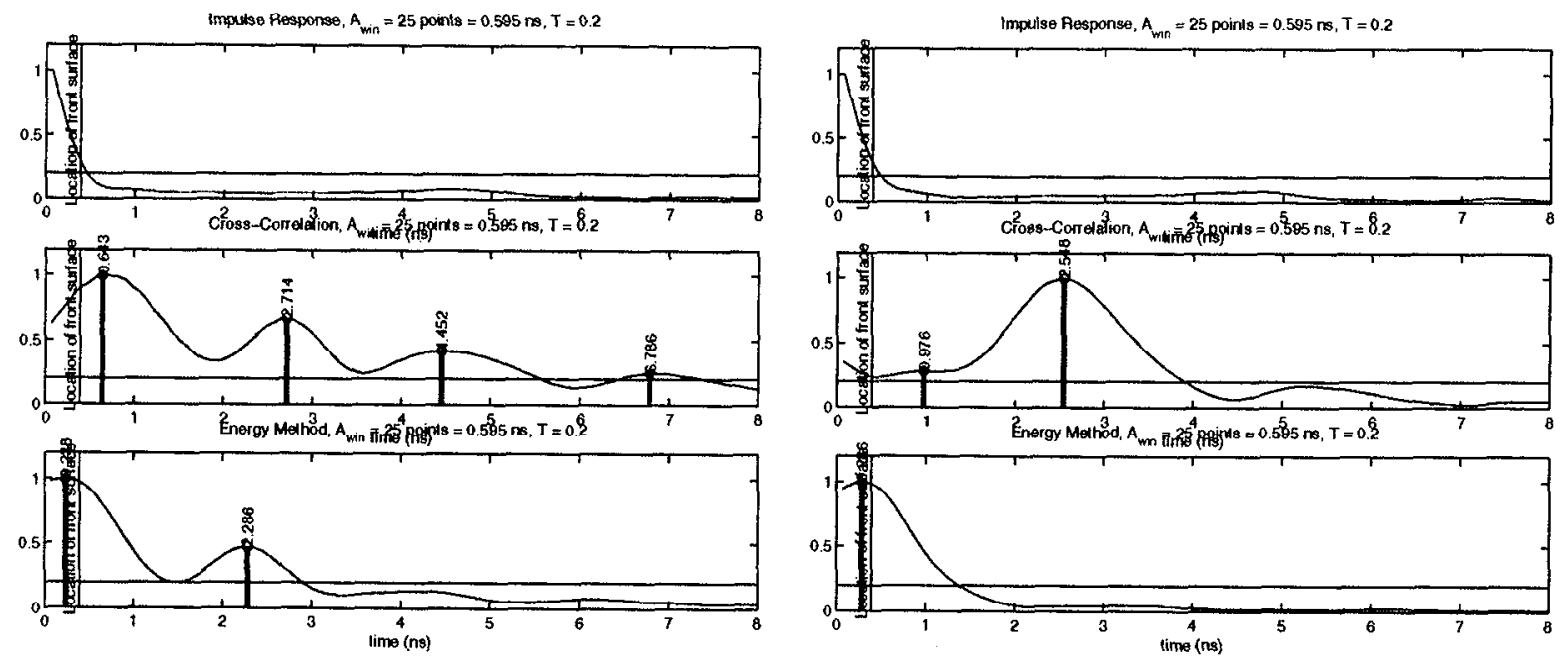

(a)

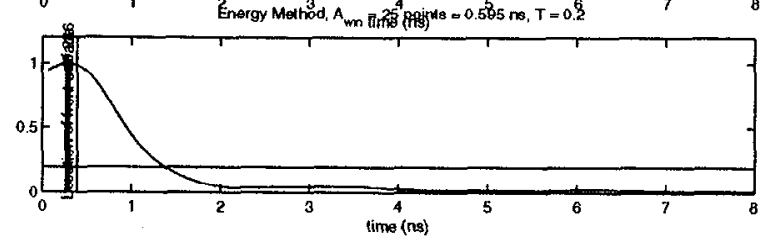

(b)
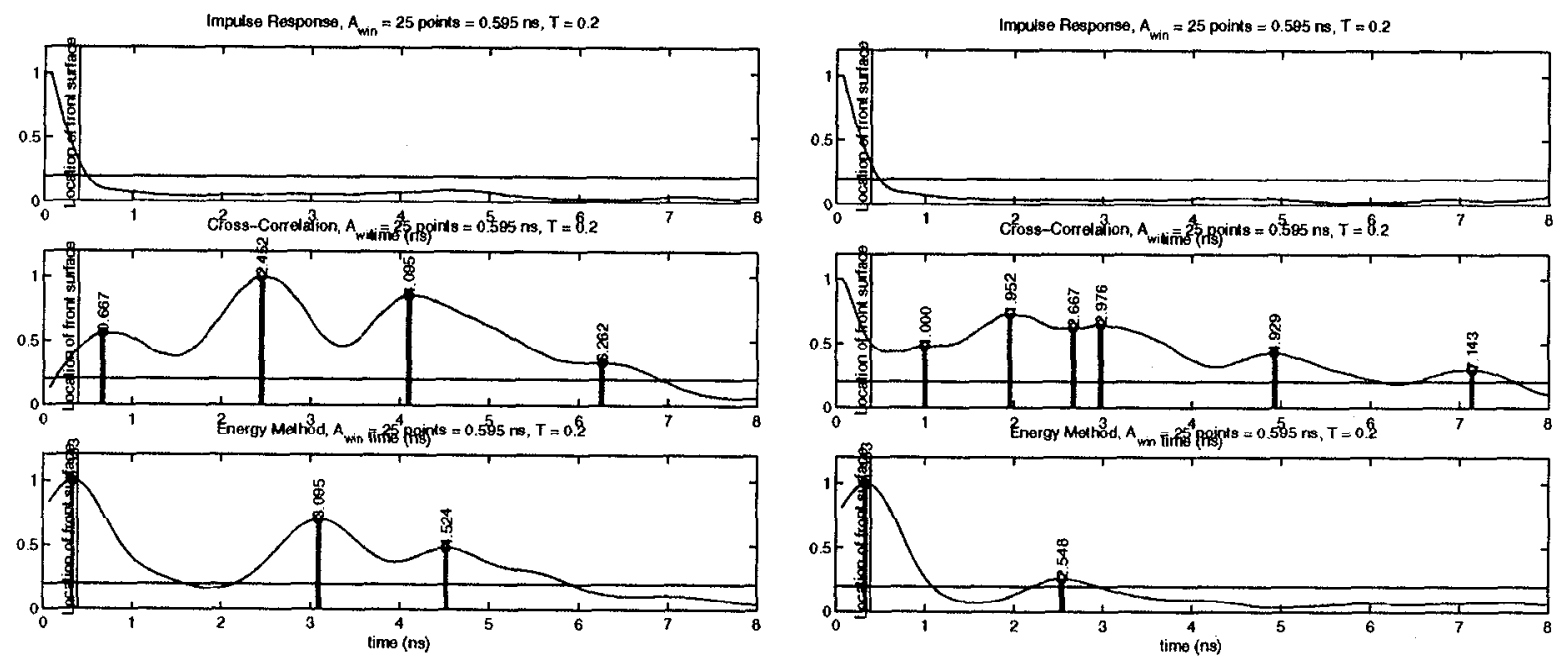

(c)

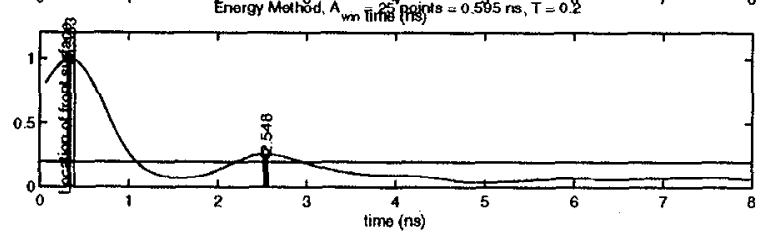

(d)

Figure 25: Results from wall number 1 in which the cinder block voids were empty. Within each group of three plots, the top is the impulse response method, the middle the crosscorrelation method, and the bottom the energy method. The four radar positions are shown in Figure 24. 

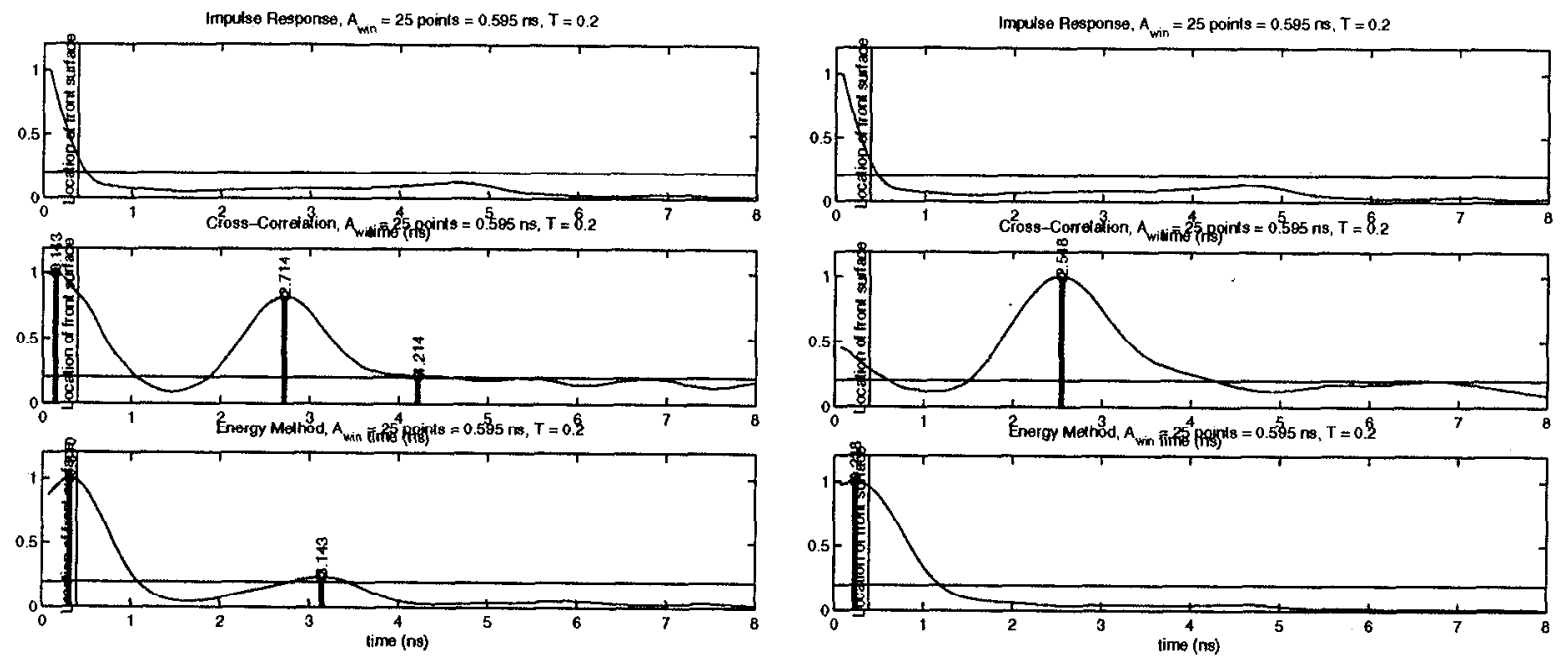

(a)

(b)
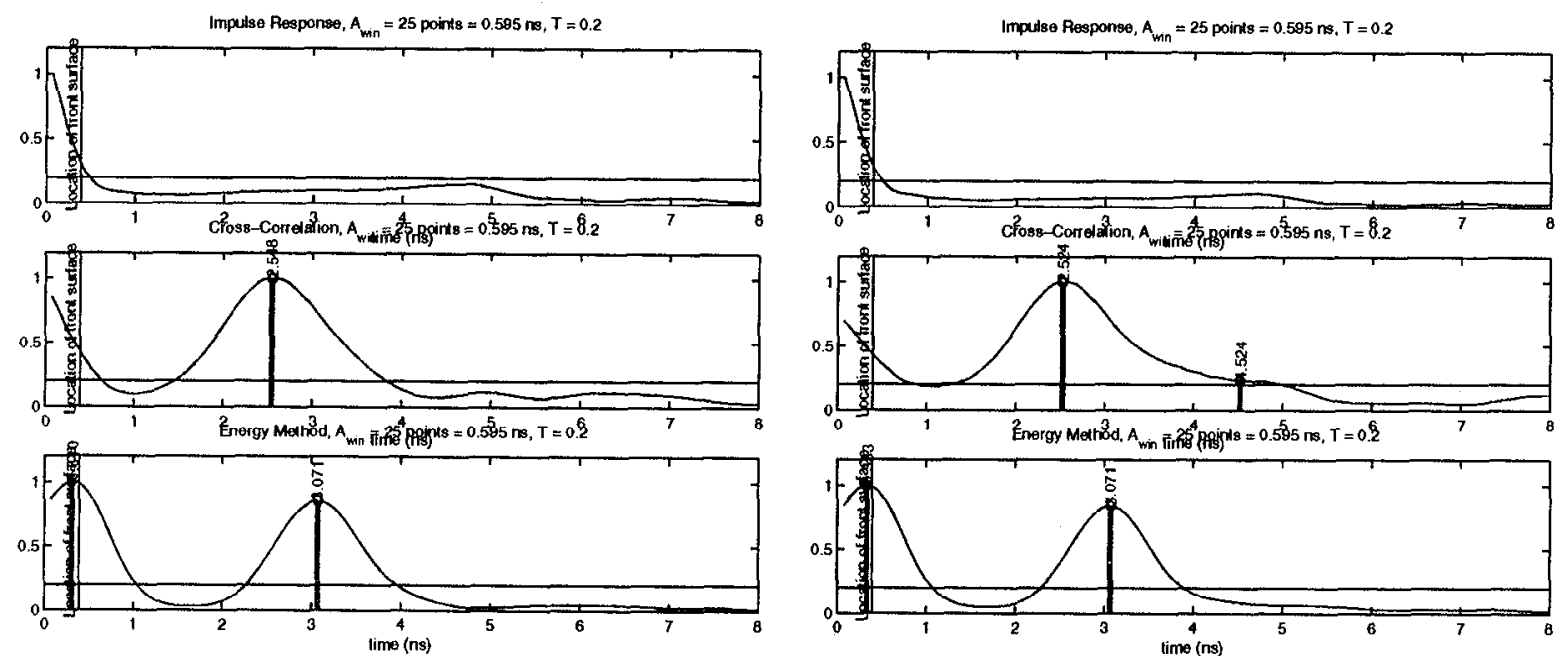

(c)

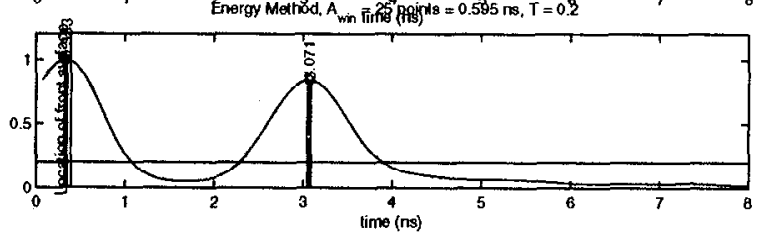

(d)

Figure 26: Results from wall number 2 in which the cinder block voids were filled with pea gravel. Within each group of three plots, the top is the impulse response method, the middle the cross-correlation method, and the bottom the energy method. The four radar positions are shown in Figure 24. 

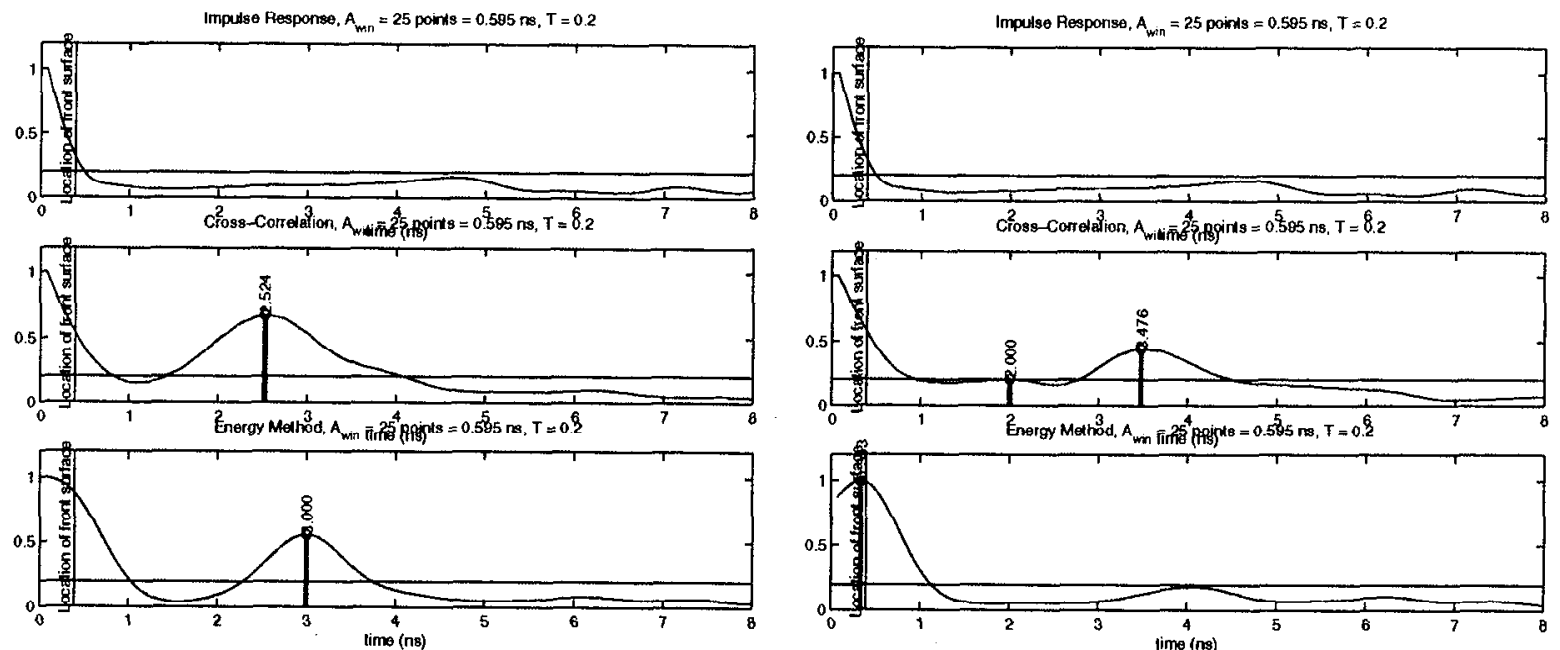

(a)

(b)
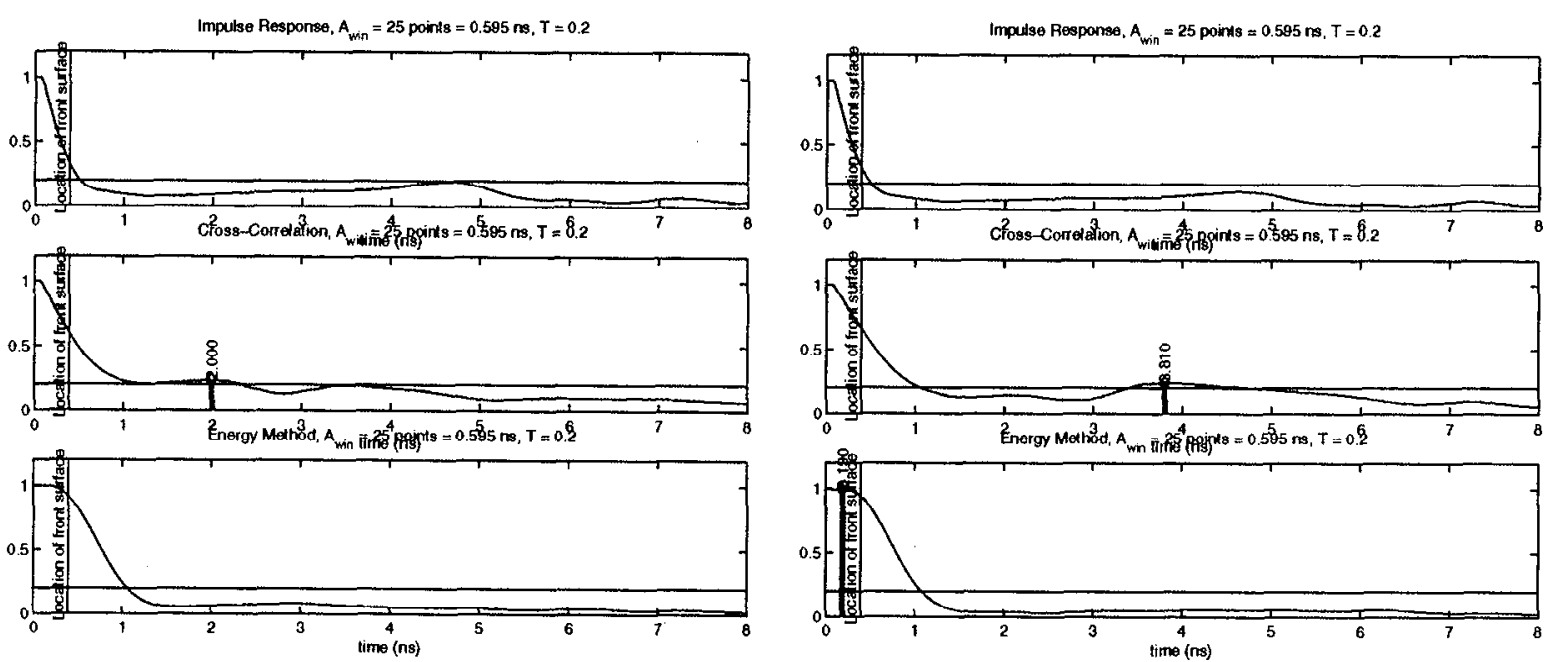

(c)

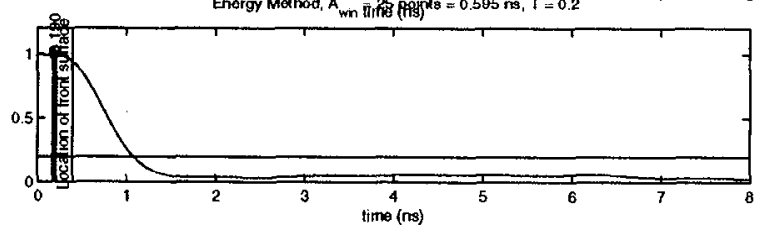

(d)

Figure 27: Results from wall number 3 in which the cinder block voids were filled with cement. Within each group of three plots, the top is the impulse response method, the middle the cross-correlation method, and the bottom the energy method. The four radar positions are shown in Figure 24. 

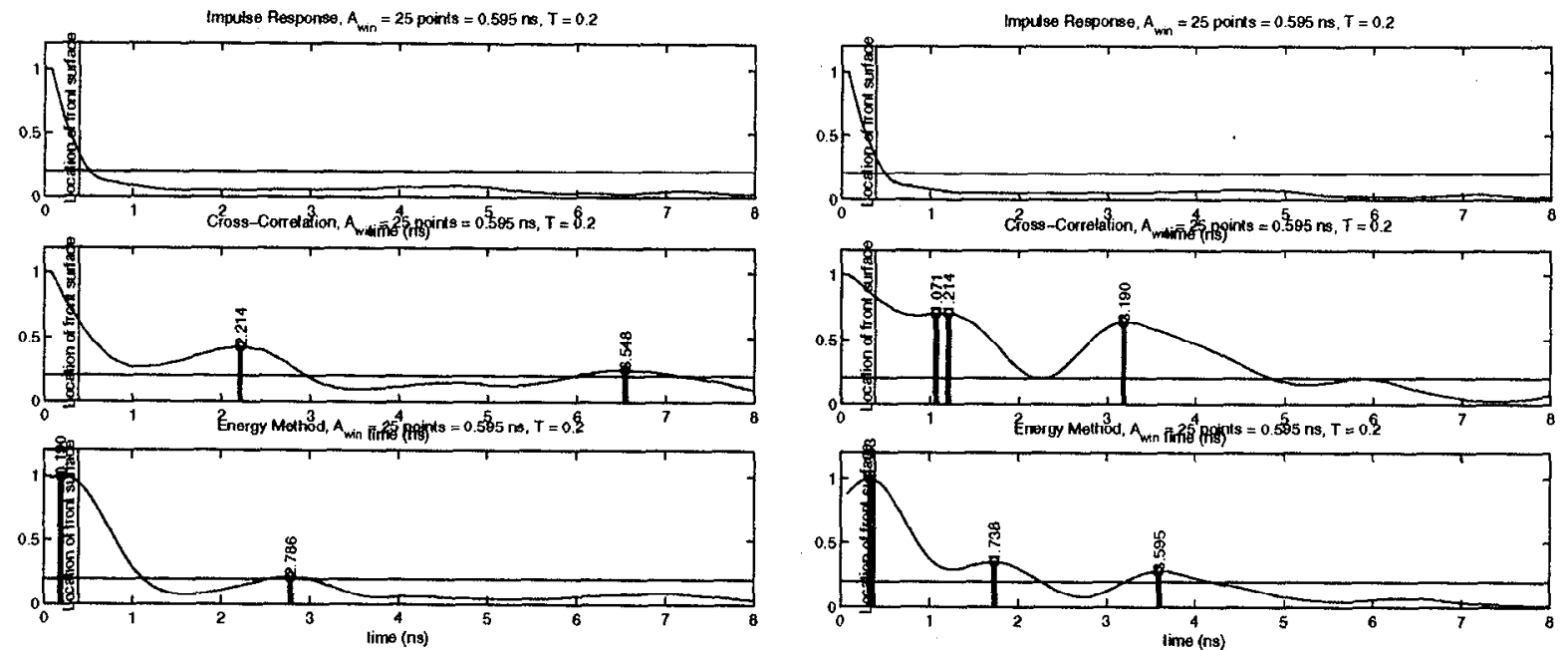

(a)

(b)
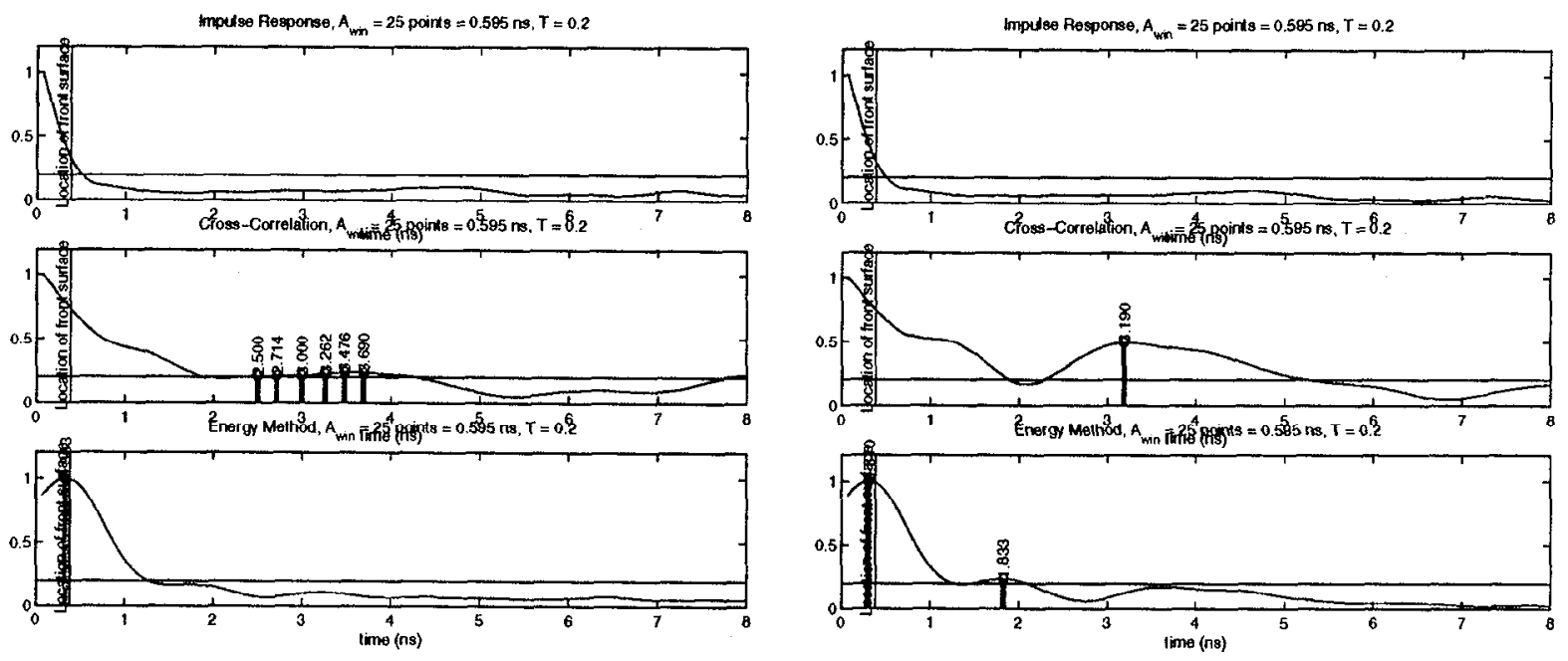

(c)

(d)

Figure 28: Results from wall number 4 in which the cinder block voids were filled with cement and a rebar down the center. Within each group of three plots, the top is the impulse response method, the middle the cross-correlation method, and the bottom the energy method. The four radar positions are shown in Figure 24. 
$18 \mathrm{~cm} \mathrm{DOG}$

\begin{tabular}{|l|llll|}
\hline Method & Layer 1 & Layer 2 & Layer 3 & Layer 4 \\
\hline Impulse Response & $5 \%$ & $4 \%$ & $15 \%$ & - \\
Cross-Correlation & $5 \%$ & $4 \%$ & - & $2 \%$ \\
Max. Like. & $3 \%$ & $2 \%$ & - & $5 \%$ \\
Roth & $1 \%$ & - & - & - \\
PHAT & $1 \%$ & - & - & $4 \%$ \\
SCOT & $1 \%$ & $4 \%$ & - & - \\
Energy & $1 \%$ & $1 \%$ & - & - \\
\hline
\end{tabular}

Ideal $18 \mathrm{~cm}$ DOG

\begin{tabular}{|l|llll|}
\hline Method & Layer 1 & Layer 2 & Layer 3 & Layer 4 \\
\hline Impulse Response & $0 \%$ & $0 \%$ & $0 \%$ & - \\
Cross-Correlation & $0 \%$ & $0 \%$ & $0 \%$ & - \\
Max. Like. & $0 \%$ & $0 \%$ & $0 \%$ & $3 \%$ \\
Roth & $0 \%$ & $10 \%$ & $16 \%$ & - \\
PHAT & $0 \%$ & $0 \%$ & - & - \\
SCOT & $0 \%$ & $0 \%$ & - & - \\
Energy & $1 \%$ & $1 \%$ & - & - \\
\hline
\end{tabular}

Table 8: Percentage error in layer detection. "- indicates the layer was not detected. 
$5 \mathrm{GHz}$ Chirp

\begin{tabular}{|c|c|c|c|c|}
\hline Method & Layer 1 & Layer 2 & Layer 3 & Layer 4 \\
\hline Impulse Response & $21 \%$ & $9 \%$ & $5 \%$ & - \\
\hline Cross-Correlation & $12 \%$ & $7 \%$ & $4 \%$ & - \\
\hline Max. Like.* & $18 \%$ & $12 \%$ & $6 \%$ & $0 \%$ \\
\hline Roth & $17 \%$ & - & - & - \\
\hline PHAT & $18 \%$ & $8 \%$ & - & - \\
\hline $\mathrm{SCOT}$ & $17 \%$ & $8 \%$ & - & - \\
\hline Energy* & $0 \%$ & - & - & $2 \%$ \\
\hline
\end{tabular}

Ideal $5 \mathrm{GHz}$ Chirp

\begin{tabular}{|c|c|c|c|c|}
\hline Method & Layer 1 & Layer 2 & Layer 3 & Layer 4 \\
\hline Impulse Response & $0 \%$ & $0 \%$ & $0 \%$ & - \\
\hline Cross-Correlation & $0 \%$ & $0 \%$ & $0 \%$ & - \\
\hline Max. Like.* & $0 \%$ & $0 \%$ & $4 \%$ & $3 \%$ \\
\hline Roth & $0 \%$ & - & - & - \\
\hline PHAT & $0 \%$ & $0 \%$ & - & $1 \%$ \\
\hline SCOT & $0 \%$ & $0 \%$ & - & $1 \%$ \\
\hline Energy & $4 \%$ & $26 \%$ & 一 & $4 \%$ \\
\hline
\end{tabular}

Table 9: Percentage error in layer detection. "*" indicates there were multiple false detections. "_" indicates the layer was not detected. 
$4 \mathrm{~cm} \mathrm{DOG}$

\begin{tabular}{|c|c|c|c|c|}
\hline \multicolumn{5}{|c|}{ DUG } \\
\hline Impulse Response & $4 \%$ & $3 \%$ & $2 \%$ & $3 \%$ \\
\hline Cross-Correlation & $0 \%$ & $2 \%$ & $1 \%$ & - \\
\hline Max. Like. & $2 \%$ & $12 \%$ & $16 \%$ & - \\
\hline Roth & $2 \%$ & - & - & - \\
\hline PHAT & $3 \%$ & $9 \%$ & - & - \\
\hline SCOT & $3 \%$ & - & - & - \\
\hline Energy & $1 \%$ & - & - & - \\
\hline
\end{tabular}

Ideal $4 \mathrm{~cm}$ DOG

\begin{tabular}{|l|lllll|}
\hline \multicolumn{7}{|c|}{ Ideal } \\
\hline Method & Layer 1 & Layer 2 & Layer 3 & Layer 4 \\
\hline Impulse Response & $0 \%$ & $0 \%$ & $0 \%$ & - \\
Cross-Correlation & $0 \%$ & $0 \%$ & $0 \%$ & - \\
Max. Like.* $^{*}$ & $0 \%$ & $0 \%$ & $4 \%$ & $0 \%$ \\
Roth* $_{\text {PHAT }}$ & $0 \%$ & $1 \%$ & - & - \\
SCOT & $0 \%$ & $0 \%$ & - & - \\
Energy & $0 \%$ & $0 \%$ & - & - \\
\hline
\end{tabular}

Table 10: Percentage error in layer detection. "**" indicates there were multiple false detections. "-" indicates the layer was not detected. 


\section{References}

[1] S. G. Azevedo and T. E. McEwan. Micropower impulse radar. Science and Technology Review, Lawrence Livermore National Laboratory, pages 16-29, 1996. UCRL-52000-96$1 / 2$.

[2] S. G. Azevedo, T. E. McEwan, and J. P. Warhus. Microradar development. Engineering Research, Development and Technology: Thrust Area Report, Lawrence Livermore National Laboratory, pages 6-17, 1996. UCRL-53868-95.

[3] A. C. Kak and M. Slaney. Principles of Computerized Tumographic Imaging. IEEE Press, 1988. ISBN 0-7803-0447-0.

[4] Lawrence Livermore National Laboratory. MIR Technology Overview, http://lasers.Ilnl.gov/lasers/idp/mir/overview.html.

[0] G. Barton. Elements of Green's Functions and Propagation - Potentials, Diffusion and Waves. Oxford University Press, 1991. ISBN 0-19-851988-6.

[6] C. A. Balanis. Advanced Engineering Electromagnetics. John Wiley \& Sons, 1989. ISBN 0-471-62194-3.

[7] W. C. Chew. Waves and Fields in Inhomogeneous Media. IEEE Press, 1995. ISBN 0-7803-1116-7.

[8] A. J. Devaney. A filtered backpropagation algorithm for diffraction tomography. Ultrasonic Imaging, 4(4):336-350, October 1982.

[9] J. E. Mast. Microwave Pulse-Echo Radar Imaging for the Nondestructive Evaluation of Civil Structures. PhD thesis, University of Illinois, Urbana-Champaign, 1993.

[10] R. W. Deming and A. J. Devaney. Diffraction tomography for multi-monostatic ground penetrating radar imaging. Inverse Problems, 13:29-45, 1997.

[11] Ronald N. Bracewell. The Fourier Transform and Its Applications. McGraw-Hill, 2 edition, 1986. ISBN 0-07-007015-6. 
[12] K. S. Yee. Numerical solution of initial boundry value problems involving Maxwell's equations in isotropic media. IEEE Transactions on Antennas and Propagation, AP14(3):302-307, May 1966.

[13] A. Taflove. Computational Electrodynamics - The Finite-Difference Time-Domain Method. Artech House, Inc., 1995. ISBN 0-89006-792-9.

[14] Gerrit Mur. Absorbing boundary conditions for the finite-difference approximation of the time-domain electromagnetic-field equations. IEEE Transactions on Eletromagnetic Compatibility, EMC-32(4):377-382, November 1981.

[15] A. V. Oppenheim and R. W. Schafer. Digital Signal Processing. Prentice-Hall, 1975. ISBN 0-13-214635-5.

[16] C. H. Knapp and G. C. Carter. The generalized correlation method for estimation of time dela. In G. C. Carter, editor, Coherence and Time Delay Estimation - An Applied Tutorial for Research, Development, Test, and Evaluation Engineers, pages 138-144. IEEE Press, 1993. ISBN 0-7803-1006-3.

[17] J. V. Candy. Signal Processing - The Modern Approach. McGraw-Hill, 1988. ISBN 0-07-100410-6. 